\title{
Distinguishing the time- and magnitude-difference accounts of the Simon effect: Evidence from the reach-to-touch paradigm
}

\author{
Matthew Finkbeiner ${ }^{1}$ - Andrew Heathcote ${ }^{2,3}$
}

Published online: 29 December 2015

(C) The Psychonomic Society, Inc. 2015

\begin{abstract}
A Simon effect occurs when the irrelevant spatial attributes of a stimulus conflict with choice responses based on non-spatial stimulus attributes. Many theories of the Simon effect assume that activation from task-irrelevant spatial attributes becomes available before the activation from taskrelevant attributes. We refer to this as the time-difference account. Other theories follow a magnitude-difference account, assuming activation from relevant and irrelevant attributes becomes available at the same time, but with the activation from irrelevant attributes initially being stronger. To distinguish these two accounts, we incorporated the responsesignal procedure into the reach-to-touch paradigm to map out the emergence of the Simon effect. We also used a carefully calibrated neutral condition to reveal differences in the initial onset of the influence of relevant and irrelevant information. Our results establish that irrelevant spatial information becomes available earlier than relevant non-spatial information. This finding is consistent with the time-difference account and inconsistent with the magnitude-difference account. However, we did find a magnitude effect, in the form of reduced interference from irrelevant information, for the second of a sequence of two incongruent trials.
\end{abstract}

Keywords Attention - Selective attention - Cognitive control $\cdot$ Automaticity

Matthew Finkbeiner

matthew.finkbeiner@mq.edu.au

1 Perception in Action Research Centre and Department of Cognitive Science, Macquarie University, Sydney, NSW 2109, Australia

2 School of Medicine, University of Tasmania, Hobart, TAS, Australia

3 School of Psychology, University of Newcastle, Newcastle, NSW, Australia

\section{Introduction}

Being fast and accurate in a choice task often requires selective attention to relevant stimulus attributes, particularly when irrelevant attributes are associated with a conflicting response. In the laboratory, choice conflict is commonly studied using variants of the Stroop (1935) task, where the irrelevant dimension is usually linguistic, and the Simon task (Simon \& Rudell, 1967), where interference arises from correspondence between the irrelevant spatial attributes of stimuli and responses. MacLeod's (1991) review of the Stroop literature endorses two models, Cohen, Dunbar and McClelland (1990) - where interference depends on the relative strengths of relevant and irrelevant stimulus attributes, and Logan (1980) - which also allows for differences in the relative speed-of-processing of relevant and irrelevant attributes. Subsequent, highly influential papers have elaborated the strength-only perspective into a general behavioral and neural theory of conflict resolution and cognitive control (e.g., Botvinick, Braver, Barch, Carter, \& Cohen, 2001; Miller \& Cohen, 2001). Theories of the Simon task, in contrast, have in the main emphasized the importance of relative speed-ofprocessing.

Hommel (1993a) proposed that the task-irrelevant spatial information in the Simon task is encoded rapidly, but that it also decays rapidly. The magnitude of interference depends on the temporal overlap between response activation caused by the transient spatial information and response activation caused by a slower and more persistent encoding of taskrelevant information. De Jong, Liang, and Lauber (1994) proposed a dual-route model (see also Frith \& Done, 1986; Kornblum, Hasbroucq \& Osman, 1990; Sanders, 1967) combining a fast direct route that transiently encodes spatial information with a second slower route that can, by itself, also be a source of interference. The slower route performs a 
transformation of the stimulus that is supposed to follow task instructions, but it can also produce interference because both relevant and irrelevant attributes are encoded (Hedge \& Marsh, 1975). This stimulus-compatibility interference broadened earlier purely stimulus-response compatibility conceptualizations, leading Hasbroucq and Guiard (1991) to characterize the Simon effect as a spatial variant of the Stroop effect. They located the stimulus compatibility effect in a stimulusidentification stage but subsequently Lu and Proctor (1994) reported evidence consistent with both compatibility effects influencing the same response-selection stage.

Dual-route architectures are now common in models of the Simon effect (e.g., Ridderinkhof, 2002a; Tagliabue, Zorzi, Umilta, \& Bassignani, 2000). The fast and slow routes are often referred to as, respectively, "direct" and "cognitive" routes, and the magnitude of Simon-effect interference is assumed to be a function of both the relative speed and strength of the two routes (Lu \& Proctor, 1995). A direct route instantiating "an initial tendency to react towards the source of stimulation" (Craft \& Simon, 1970, p.415) has strong face validity. This is particularly so in the most commonly used "horizontal" version of the Simon task - where the stimuli and response keys are located on the left and right - given the tendency to reach for a source of lateral stimulation with the corresponding hand that is learned early in life and is perhaps to some degree innate (Umilta \& Zorzi, 1997).

The primary aim of the present study is to determine whether, as is usually assumed, activation from taskirrelevant attributes becomes available earlier than activation from task-relevant attributes. We will refer to this possibility as the "time-difference account." Alternatively, it is equally possible that activation from both relevant and irrelevant attributes become available at the same time, but with the activation from irrelevant attributes initially being stronger. We will refer to this as the "magnitude-difference account." The a priori plausibility of the time difference account can be validated by consulting the neuroanatomical paths supporting the processing of the different attributes and independently tested, such as by measuring simple reaction times to each, but the same cannot be said for the magnitude approach. Our analysis aims to address this imbalance.

Figure 1 schematically depicts the magnitude-difference and time-difference explanations for conflict trials, where positive values indicate activation favoring the correct response (see Ivanoff, Klein, \& Lupianez, 2002, and Ivanoff, 2003, for related depictions of models of the time course of the factors underlying the Simon effect). Critically, the total activation, which is the sum of relevant and irrelevant activation, is similar in both cases, varying between -1 and 1 , demonstrating that both explanations yield the same (correct) predictions for the time course of the conflict effect. This is especially true for measures, like response latencies or accuracy rates, insofar as both accounts correctly predict larger interference effects in the earliest responses and attenuated interference effects in later responses. Thus, to date it has been very difficult to distinguish the time- and magnitude-difference accounts of the Simon effect. Fortunately, it is possible to adjudicate these two accounts by including a neutral condition in a task that incorporates a measure that can reveal the emergence of the Simon effect over time.

To distinguish the time- and magnitude-difference accounts, one needs to distinguish the relative differences in time between when the task-relevant and task-irrelevant information first begin to influence the behavioral response. To isolate the influence of task-relevant information, we use a neutral stimulus, which is presented along the vertical meridian and, therefore, does not carry with it any task-irrelevant spatial information. We then take advantage of the temporal resolution afforded by the reach-to-touch paradigm (Buetti \& Kerzel, 2008, 2009; Finkbeiner, Coltheart \& Coltheart, 2014) to identify the points in time that the task-relevant and taskirrelevant spatial information first begin to influence the response. If these are the same points in time, the magnitudedifference account should be favored. If, though, one finds that the task-irrelevant spatial information begins to influence the response earlier than the task-relevant information, the time-difference account should be favored. In what follows, we take advantage of the ability of the reach-to-touch paradigm to reveal the emergence of experimental effects across time to identify the points in time that the task-relevant and irrelevant information first begin to influence the response. To anticipate our results, our findings support the time-difference account - insofar as we find that the task-irrelevant spatial position of the target stimulus begins to influence responses before the task-relevant information does - but that a magnitude-difference account has a role in modulation of the Simon effect by trial history (i.e., sequential effects, Hommel, Proctor, \& Vu, 2004; Stürmer et al., 2002).

In the next section we examine three major sources of evidence about the time course of interference in the Simon task: (1) manipulations of discriminability and stimulus-onset asynchrony that affect the time at which relevant and irrelevant information becomes available, (2) differences in the magnitude of interference for fast and slow responses, and (3) hemispheric asymmetries in evoked response potentials (ERPs). We then report the results of two experiments that use quantitative characteristics of reaching movements to provide a finegrained characterization of the time course of the influence of relevant and irrelevant information in the Simon effect.

\section{The Simon effect}

The Simon effect was first quantified in terms of an increase in mean error rates and response time (RT) for incongruent trials (e.g., a stimulus requiring a left-hand response presented on 
Magnitude Difference

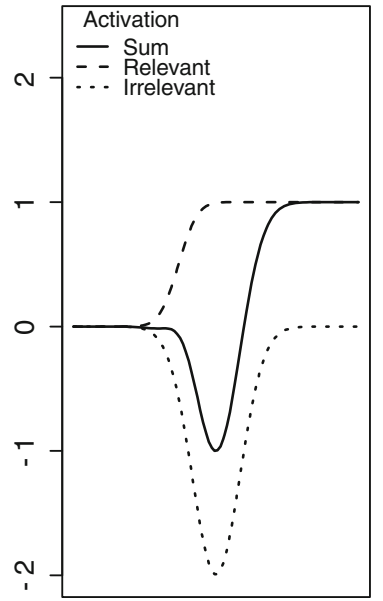

Time Difference

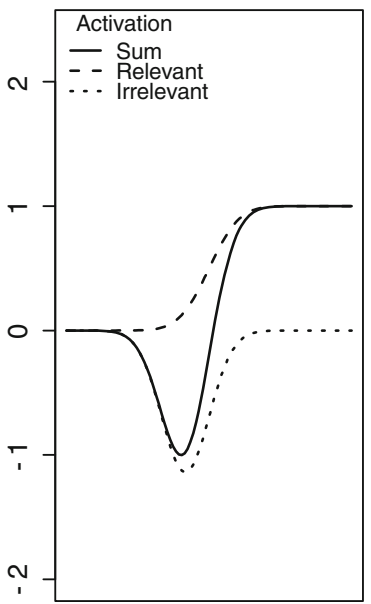

No Decay

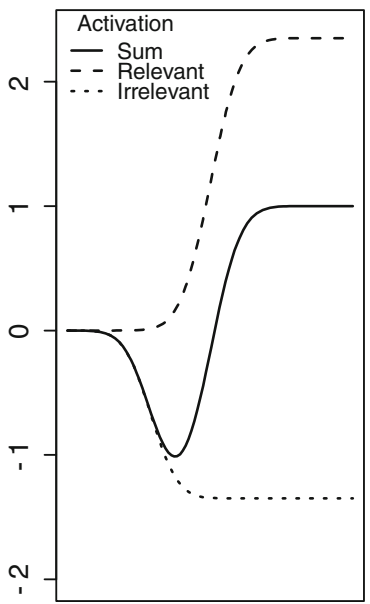

Fig. 1 Activation (y-axis) associated with irrelevant and relevant information, and their sum, as a function of time (x-axis) for conflict trial. Positive activation values indicate support for the correct response. In the left panel relevant and irrelevant information diverge from baseline at the same time but the irrelevant information is initially bigger. In the middle panel the irrelevant information diverges from baseline earlier than the relevant information, with both attaining the same peak magnitude, but the irrelevant information also decays whereas the relevant information maintains its peak. In the right-most panel they again diverge at different times but both maintain their peak magnitude. This peak is higher for the relevant information and so the same biphasic shape occurs in the sum. Note that the early summed activity away from the correct response will likely have a peak magnitude less than the later summed activity toward the correct response, but is shown as equal in order to make it easier to contrast the time and magnitude difference accounts the right) relative to congruent trials (e.g., a stimulus requiring a left hand response presented on the left). In Simon and Rudell's (1967) original study auditory stimuli were presented either on the left or right and participants were instructed to press a button with their left hand when they heard the word "left" and press a button with their right hand when they heard the word "right." Interference can be defined as a positive quantity corresponding to RT or error rates for congruent trials subtracted from the corresponding measure for incongruent trials. Interference occurs both when stimulusresponse associations are pre-existing, as in Simon and Rudell's study, and also when they are defined by task instructions (e.g., left and right responses to, respectively, high and low tones, Simon \& Small, 1969, or red and green patches, Hedge \& Marsh, 1975).

Simon, Acosta, Mewaldt, and Speidel (1976) investigated the time course of the Simon effect by delaying responding. Their first experiment used what is now referred to as a "standard" design (Proctor Miles \& Baroni's, 2011), with responses made by either the left or right hand to visual stimuli presented on either the left or right. However, it added an extra requirement, that participants delayed their response until they heard a tone that could occur at durations of $0 \mathrm{~ms}, 150 \mathrm{~ms}$, $250 \mathrm{~ms}$, or $350 \mathrm{~ms}$ after the visual stimulus. The Simon effect was eliminated for the latter two intervals (see also Burle, van den Wildenberg \& Ridderinkhof, 2005; Vallesi \& Umiltà, 2009; Wiegand \& Wascher, 2005; but see Ivanoff, 2003 for a different pattern when the cue could also indicate that the response be withheld). A second experiment using high- and low-tone stimuli sought to directly control when response selection could begin. Participants were either given the same stimulus-response mapping on all trials or the mapping was varied, being specified on each trial $1 \mathrm{~s}$ before, simultaneously with, or $150 \mathrm{~ms}, 250 \mathrm{~ms}$, or $350 \mathrm{~ms}$ after the visual stimulus. The Simon effect disappeared for the latter three intervals. Simon et al. concluded that interference affects the response selection stage, but that it persists for only around $250 \mathrm{~ms}$.

Several authors have shown that the Simon effect is reduced by manipulations that slow discrimination of the relevant attribute. Hommel (1993a) found a reduced effect when he slowed discrimination through greater retinal eccentricity, reduced contrast, adding overlaid patterns, or gradually building up the stimulus over time. Hommel (1994a) found the Simon effect completely disappeared for a difficult square versus rectangle discrimination, but was present in the same paradigm for an easier color discrimination. Roswarski and Proctor (1996) compared color, circle versus Square, and rectangle versus square discriminations that took increasingly longer times to perform, and found an associated decrease in the Simon effect. Vallesi and Umiltà (2009) also found that a decrease in the standard Simon task when the difficulty of a color discrimination was increased. All of these results are consistent with the idea that the slowed discrimination reduces the Simon effect by decreasing the temporal overlap of activation in the cognitive route and the early occurring - but then quickly reducing - activation in the direct route. Ivanoff, Klein, and Lupianez (2002) reported an apparent exception to this pattern, where the Simon effect was 
increased when responding was slowed by an inhibition of return (IOR: Taylor \& Klein, 1998). However, this was attributed to IOR producing a large increase in interference (by delaying, increasing, or slowing the decay of activation in the direct route) rather than as evidence against the overlap hypothesis.

De Jong et al.'s (1994) seminal paper proposed a more finegrained analysis of the Simon effect than is provided by mean RT, by quantifying interference as a function of overall response speed. They introduced the delta function (a term coined later by Ridderinkhof, 2002b), which is created by calculating a set of quantiles (e.g., deciles) for incongruent and congruent RT distributions and then plotting the difference between corresponding values (see Speckman, Rouder, Morey, \& Pratte, 2008, for technical discussion). De Jong et al. found decreasing delta functions (i.e., a larger interference effect for fast than for slow responses), which they interpreted as indicative of a tendency to respond toward the source of stimulation that arose quickly after stimulus onset but which also dissipated quickly. They speculated that dissipation might be due to either passive decay or active suppression. Subsequently, support has been reported for both the decay (e.g., Hommel, 1994b) and suppression (e.g., Ridderinkhof, 2002a, 2002b; Forstmann, van den Wildenberg, \& Ridderinkhof, 2008) accounts.

De Jong et al. (1994) came to the strong conclusion that "the results of the distributional analyses of spatial SRC [Simon] effects in the experiments reported here almost certainly provide a reliable estimate of the actual time course of these effects" (p.733). Burle et al. (2005) provided converging evidence for this claim, finding that delta function slopes were strongly correlated with changes in the Simon effect due to a direct manipulation of the relative times at which location and colour information became available. However, Zhang and Kornblum (1997; see also Roswarski \& Proctor, 2003) challenged De Jong et al.'s conclusion. They pointed out that delta-function slopes could simply be due to differences between incongruent and congruent RT variance. If the slower incongruent responses are also less variable than the faster congruent responses, a decreasing delta function results. Zhang and Kornblum provided a detailed analysis of Kornblum's (1994) Simon-effect data where variance was larger for incongruent than congruent conditions and the slope of the delta function was positive. They also pointed to a variety of other data sets where the ordering of congruent and incongruent variances was inconsistent.

In addition to these cued versions of the Simon task, there have been numerous reports with un-cued Simon tasks where the Simon interference effect does not decrease with increasing RTs, such as in a horizontal Simon task with auditory stimuli and also with visual stimuli and response hands crossed (Wascher, Schatz, Kuder, \& Verleger, 2001), and when responses and visual stimuli are presented in a vertical arrangement (Wiegand \& Wascher, 2005). To explain the different patterns of results obtained with horizontal and vertical displays, Wascher et al. (2001) proposed that the fast direct route corresponds to a specialized brain network associated with grasping that is only active with visual stimuli presented horizontally and bi-manual uncrossed responding (i.e., the standard Simon task). Wiegand and Wascher (2005, 2007) refined this position to address decreasing delta function found with uni-manual (i.e., different fingers on the same hand) responding, and vertical presentations with variable response rules. However, controversy surrounds Wascher et al.'s (2001) original explanation (cf. Roswarski \& Proctor, 2003) and subsequent developments of it, with Proctor et al. (2011) saying that these developments need further testing.

The dual-route theories proposed by both De Jong et al. (1994) and Wascher et al. (2001) have also drawn support from ERPs, and in particular the lateralized readiness potential (LRP), which is calculated from the average asymmetry in ERPs over left and right primary motor cortex. In a vertical Simon task, De Jong et al. found that the LRP on congruent trials reached significant amplitudes at $170 \mathrm{~ms}$ after stimulus onset. On incongruent trials, they observed an initial asymmetry corresponding to the wrong choice in the same time frame. This initial asymmetry or "LRP dip" (Leuthold, 2011) in the wrong direction on incongruent trials generally reaches significant amplitudes in the same time window (or slightly later) as the LRP in the correct direction on congruent trials (see also Stürmer, Leuthold, Soetens, Schröter, \& Sommer, 2002; Valle-Inclán, 1996). While this finding is clear in establishing that the task-irrelevant spatial information is able to gain control of the response formulation process, we are not aware of any LRP studies that have attempted to distinguish between the time- and magnitude-difference accounts. In fact, we are aware of only one LRP study that tried to quantify the response to the task-relevant source of information in isolation (Cespón, Galdo-Álvarez, \& Díaz, 2012). Somewhat surprisingly, Cespón and colleagues found in this study that the LRP for incompatible trials (task-irrelevant information) was delayed relative to the LRP for neutral trials (task-relevant information). Taking this result at face value, it would appear to go against the widely-held view that task-irrelevant information gains control of the response formulation process earlier than the task-relevant information.

It is not clear at this point how this result should be interpreted, but we note that the neutral stimuli were presented at fixation in this study, meaning that the neutral stimuli were potentially better attended than the peripherally presented stimuli. Furthermore, and more generally, it is well known that the LRP dip in the horizontal Simon task is very difficult to disentangle from early sensory ERP components associated with laterally presented stimuli (Praamstra, 2007). Indeed, Leuthold (2011) concluded that further work is required to clarify the conclusions that can be drawn from findings about 
an LRP dip given the inconsistent results for vertical Simon task and the potential for confounds by sensory components in the horizontal Simon task. In the present paper, we use statistics measured from reaching movements in a horizontal Simon task to provide the same sort of fine-grained temporal analysis of the time course of the Simon effect afforded by electroencephalograms (EEGs) while avoiding the confounds noted by Praamstra and Leuthold.

\section{Reach-to-touch paradigm}

The reach-to-touch paradigm is becoming an increasingly popular tool for cognitive scientists. Reaching trajectories offer researchers a continuous behavioral measure that promises to reveal the unfolding of decision processes with much better temporal resolution than standard behavioral measures. Not surprisingly, the reach-to-touch paradigm has already been used to investigate temporal properties of the Simon task. For example, Buetti and Kerzel $(2008,2009)$ had subjects perform the Simon task by reaching out and touching one of two response regions, depending on the color of the target stimulus. In their studies they used "initial-movement angle" as their dependent measure. Initial-movement angle is calculated by taking the angle between the linear path connecting the start and end positions and the position of the hand at the point corresponding to $20 \%$ of the total distance traveled. Buetti and Kerzel argued that the initial-movement angle reveals important details about the motor plan that is assembled just prior to movement initiation.

Buetti and Kerzel $(2008,2009)$ found an average deviation in the initial-movement angle towards the incorrect response for incongruent trials relative to congruent trials. Consistent with the decreasing delta function reported by De Jong et al. (1994), the interference effect in the initial-movement angle decreased as the period of time between stimulus onset and the movement initiation increased. We will refer to this period of time as "movement initiation time" (MIT). More specifically, they found the distribution of initial movement angles to be bimodal in the incongruent (but not congruent) condition for trials with the fastest MITs (first $20 \%$ of the MIT distribution). In contrast, the distribution of initial movement angles was unimodal for trials with longer MITs in both congruent and incongruent conditions. This finding suggests that the direction of the initial reaching response on some trials was strongly affected by the location of the target stimulus, but only if the reaching movement was initiated relatively soon after stimulus onset.

Although these findings from the reach-to-touch paradigm are encouraging in their consistency with the RT data, we note that Buetti and Kerzel (2008, 2009; see also Kerzel \& Buetti, 2012) consistently observed strong Simon effects in subjects' fastest set of responses (first quantile). As a result, the findings reported by Buetti and Kerzel are very similar to the RT effects reported elsewhere in that they reveal a strong Simon effect early on in stimulus processing followed by a weak to nonexistent effect later on, but fail to reveal the gradual emergence of the effect. To distinguish the time- and magnitudedifference accounts, one needs to identify when the taskrelevant and irrelevant information first begins to influence responses. Thus, the goal of the present study was to take full advantage of the temporal resolution offered by the reach-totouch paradigm to establish when in stimulus-processing time (i.e., relative to stimulus onset) the Simon effect first emerges.

The potential of reaching movements to reveal the realtime unfolding of mental processes hinges on their co-occurrence. That is, a reaching movement can only reasonably be thought to provide a real-time window on internal cognitive processes if it is made while those processes are still ongoing. To achieve this end, in the present study we made use of the response-signal procedure (Finkbeiner et al., 2014; Ghez, Favilla, Ghilardi, Gordon, Bermejo, \& Pullman, 1997; Reed, 1973, 1976), whereby subjects are trained to initiate their movement within a $300-\mathrm{ms}$ wide response window that "opens" $100 \mathrm{~ms}$ before an imperative go signal and "closes" $200 \mathrm{~ms}$ after the go signal. The imperative "go" signal is the final beep in a sequence of three beeps (cf. Kello \& Plaut, 2000 for a similar procedure). Importantly, we positioned the train of three beeps on each trial so that the final "go" beep was presented at specific points in time relative to the onset of the target stimulus. In this way, we elicited reaching responses that commenced across a range of stimulus viewing times, from before target onset to $\sim 400 \mathrm{~ms}$ after target onset. Note that the response-signal procedure alone with a button-press response is unable to measure early effects, as stated by Hilchey, Ivanoff, Klein, and Taylor (2011 see also Ivanoff, Blagdon, Feener, McNeil, \& Muir, 2014): "In this and other efforts ... we have been unable to get participants to respond much earlier than about $250 \mathrm{~ms}$ post target. Thus, we have been unable to visualize information processing dynamics reflecting the hypothesized accumulation of the irrelevant location information." (p. 240)

In terms of the dependent measure, ours is very similar to the initial movement angle used by Buetti and Kerzel (2008, $2009)$, but differs in that we use the mean lateral or " $x$ " velocity (see Fig. 2 for the orientation of the $\mathrm{x}$-axis in our paradigm) calculated across the first $150 \mathrm{~ms}$ of the reaching response (cf. Finkbeiner, et al., 2014; Quek \& Finkbeiner, 2013; 2014). Similar to Buetti and Kerzel's use of the $20 \%$ point in the movement, the reason for limiting the dependent measure to the initial segment of the trajectory is because the initial segment of the response is informative of the motor plan that participants had assembled just prior to movement initiation. To establish the time course of the Simon effect, we grouped the reaching movements according to when they were initiated relative to the onset of the target stimulus (MIT) and 


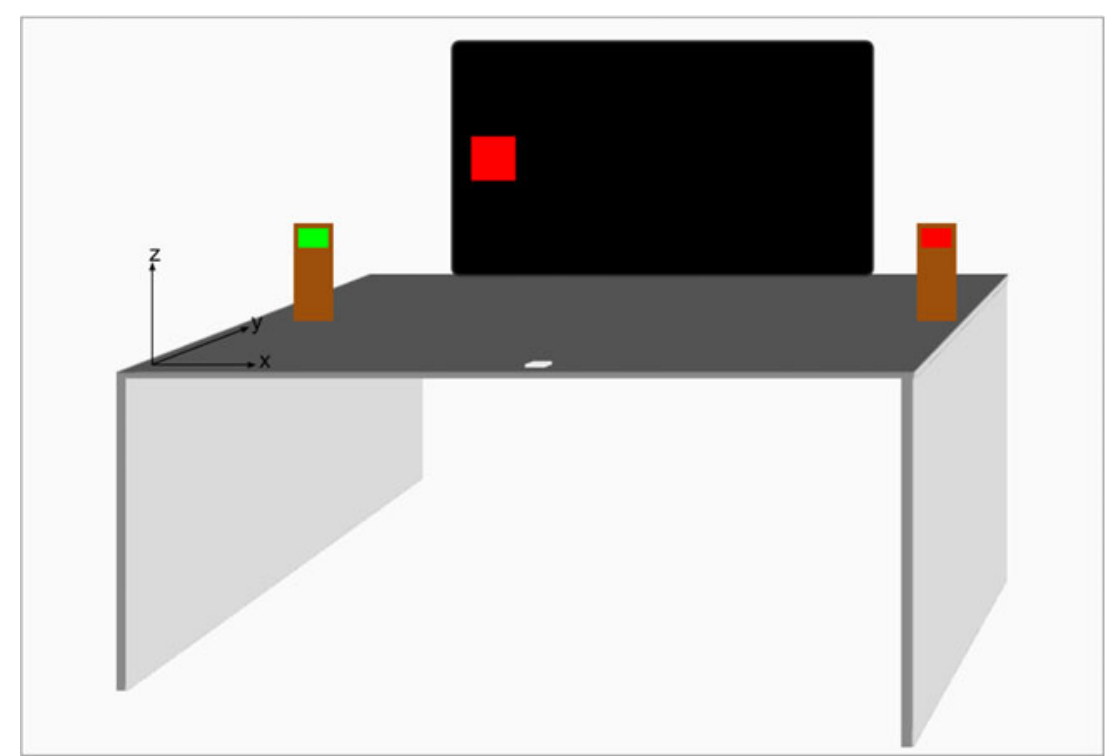

Fig. 2 Apparatus. Subjects sat at a desk with a CRT monitor and two lateral response panels. Subjects placed their right index finger at the start position (white square) to initiate the trial and then responded by reaching

analyzed the initial $\mathrm{x}$-velocities as a function of this period of target viewing time. As we demonstrate, this analysis allows us to map out the onset, growth, and subsequent decrease of the Simon effect in stimulus-processing time. To anticipate our results once again, we find that subjects' movements early on in stimulus processing (0 200 ms) are strongly influenced by the stimulus' task-irrelevant spatial information but not its task-relevant properties. In contrast, movements initiated $>200 \mathrm{~ms}$ after stimulus onset reveal how the task-relevant information begins to gain control of the responses. Taken together, this pattern of results is consistent with the timedifference account and presents a serious challenge to the magnitude-difference account.

\section{Experiment 1}

In Experiment 1, subjects were instructed to respond to the meaning of a target stimulus (the word "left" or "right") while ignoring its position. ${ }^{1}$ Subjects responded by reaching out and touching the appropriate response panel that was positioned to the left or right of the computer monitor (see Fig. 2). Note that the monitor was far enough behind the response panels (see "Apparatus" section below) that participants were able to

\footnotetext{
${ }^{1}$ Except for the inclusion of a neutral condition in our task, we note that our design closely follows the "Simon instruction condition" of Experiment 2 in Pratte, Rouder, Morey, and Feng (2010). This particular version of the Simon task was originally used by O'Leary and Barber (1993). As noted by Pratte et al., this task design corresponds to Type 8 in the Dimensional Overlap taxonomy provided by Kornblum, Stevens, Requin, and Whipple (1999) insofar as there is overlap across both stimulus-response and stimulus-stimulus dimensions.
}

out and touching one of the two response boards. An incongruent trial from Experiment 2 is depicted in which a red target presented on the left requires a response to the right

reach out and touch both response panels without their arm obscuring the monitor. The target stimuli were presented at one of four positions, at equal eccentricities, either to the left or right or above or below the center of the screen. Left and right positioned targets constituted the congruent and incongruent conditions. The stimuli presented above and below fixation constituted the neutral condition.

A neutral condition was included to provide a baseline that enabled us to establish at what points in time the different stimulus attributes (word meaning vs. position) begin to affect the decision process. We did not present a neutral stimulus at fixation as in some earlier studies (e.g., Cespón, et al., 2012; Wiegand \& Wascher, 2005) to avoid confounding the neutral condition, and hence differences between it and the congruent or incongruent condition, with the effects of a difference in eccentricity.

\section{Methods}

\section{Subjects}

Eighteen undergraduates (12 female) from the psychology subject pool at Macquarie University participated in return for course credit. All subjects had normal or corrected-tonormal vision and all reported being right-handed.

\section{Apparatus}

Subjects sat at a desk with a CRT monitor placed $75 \mathrm{~cm}$ from the front edge of the desk (see Fig. 2). Two lateral response panels $(30 \mathrm{~cm} \times 9 \mathrm{~cm})$ were positioned $50 \mathrm{~cm}$ from the front of the desk, one on the left edge of the desk, and one on the right 
edge. The desk was $75 \mathrm{~cm}$ wide. Subjects initiated a trial by moving their right index finger to the "start" position, which was located in the middle of the desk and close to its front edge. To respond, subjects reached out and touched the appropriate response panel; the left response panel for the target word LEFT, and the right response panel for the target word RIGHT.

We used a Polhemus Liberty $(240 \mathrm{~Hz})$ motion capture system with a small sensor taped to the tip of the subject's right index finger to record the subject's movements during the experiment. The advantage of using a motion-capture device, as opposed to a computer mouse (e.g., Freeman, Ambady, Rule, \& Johnson, 2008; Koop \& Johnson, 2011), is that it allows subjects to move freely and naturally in three dimensions. This is important to obtain movements with bell-shaped velocity profiles that are typical of natural movements and which are critical to the analyses that we report below.

Subjects wore headphones (Sennheiser 280 Pro), which we used to present the three beeps guiding response initiation, the final of which constituted the go signal. In Experiment 1 we used two different stimulus-onset asynchronies (SOA) between the target stimulus and go signal: $0 \mathrm{~ms}$ and $300 \mathrm{~ms}$ relative to the onset of the target. As mentioned, subjects were asked to initiate their response within a 300 -ms wide window that encompassed the go signal. If subjects did not initiate their movement within the response window, the trial was terminated with a buzz and the appropriate visual feedback (e.g., "Too Early!" or "Too Late!") was presented. Trials that were terminated due to a response window failure were re-presented at a later point in the experiment. Upon initiating a movement, subjects were required to maintain a continuous forward movement over the first 50 recorded samples ( $208 \mathrm{~ms})$ and trials were terminated with a buzz and visual feedback if this criterion was not met.

\section{Design and procedure}

Each trial began with a fixation cross for $500 \mathrm{~ms}$ at which point the sequence of three beeps began. The final go beep was presented 1,200 ms after the onset of the fixation cross, either simultaneously with the target stimulus ( $0-\mathrm{ms} \mathrm{SOA})$ or $300 \mathrm{~ms}$ after the target stimulus (300-ms SOA). The target stimulus appeared in one of four different positions (right, top, left, bottom), with the innermost edge $6^{\circ}$ from fixation. The two target words (LEFT, RIGHT) were fully crossed with the three levels of Trial Type (Congruent, Incongruent, Neutral), to yield six different Trial-Type $\times$ Target-Word combinations. These six combinations were repeated eight times per block for a total of 48 trials per block. The target appeared above fixation on half of the neutral trials and below fixation on the other half of neutral trials. Thus $33 \%$ of the trials were congruent (e.g., the word LEFT presented on the left), $33 \%$ were incongruent (e.g., the word LEFT presented on the right), and $33 \%$ were neutral (e.g., the word LEFT presented at the top or bottom position). The experiment began with two blocks of practice trials $(\mathrm{N}=96)$, followed by ten blocks of experimental trials $(\mathrm{N}=480)$.

\section{Data analysis}

Perhaps the most common way to analyze reaching trajectories is to calculate the "path offset" or "curvature" of the trajectory (Atkeson \& Hollerbach, 1985; Smit \& van Gisbergen, 1990). However, this dependent measure relies on movement corrections that occur relatively late within the reaching movement and, hence, is relatively insensitive as a measure of how responses systematically vary with stimulusviewing times. Because our primary interest was in establishing how the Simon effect unfolds during the course of stimulus processing, our approach is different. As we alluded to above, we couple the response-signal procedure with an anal$\mathrm{ysis}$ of the initial $\mathrm{x}$-velocity, which reveals information about the motor plan that participants had assembled just prior to movement initiation. We then employ a distribution analysis whereby we bin the trajectories according to their MIT to see how the Simon effect emerges over stimulus viewing time.

Note that in a small percentage of trials, the MIT value is negative due to subjects having initiated their movement within the $100 \mathrm{~ms}$ of the response window that precedes target onset (this occurs only on 0-ms SOA trials). Having a small percentage of trials with negative target-viewing times is intended. Because the initial segment of movements that are initiated prior to target onset should not reveal any experimental effects, these trials provide a baseline.

To calculate $\mathrm{x}$-velocity, we first filtered the position data with a two-way low-pass Butterworth filter at $7 \mathrm{~Hz}$ and then we calculated the derivatives (velocity, acceleration) through numerical differentiation. We then identified the movement's onset and offset. Movement onset was defined as the first of 20 consecutive samples that exceeded $10 \mathrm{~cm} / \mathrm{s}$ in tangential velocity ${ }^{2}$; movement offset was defined as the first of 20 consecutive samples that occurred after peak velocity and that fell below $10 \mathrm{~cm} / \mathrm{s}$ in tangential velocity. We then used a modified orthogonal polynomial trend analysis (OPTA) to improve the signal to noise ratio (cf. Woestenburg Verbaten, Van Hees, and Slangen's (1983; see also Karayanidis Provost, Brown, Paton, $\&$ Heathcote, 2011). In our modified version of this approach (cf. Finkbeiner et al., 2014; Quek \& Finkbeiner, 2014), the MITs for each trial are used as the only covariate in a polynomial regression model of the observed x-velocity profiles. With this approach, one can use the regression model to

\footnotetext{
2 Tangential velocity is the "speed" of the hand regardless of direction. Tangential velocity is always positive. Note that we only use tangential velocity to establish movement onset. The dependent measure is initial $\mathrm{x}$ velocity, which is a signed value.
} 
analyze the x-velocity profile for any given MIT, allowing for a very fine-grained analysis of changes in the reaching response as a function of target-viewing time. Because all trials from each cell of the experimental design contribute to the regression model, this approach is very effective in increasing the signal-to-noise ratio. For example, Woestenburg et al. (1983) reported an increase in signal-to-noise ratio over simple averaging and Wiener filtering by up to a factor of ten and Karayanidis et al. (2011) found that OPTA improved the signal-to-noise ratio by 2.5 times over simple averaging. In our OPTA procedure, trials within each individual design cell were ordered by MIT latency from 1 to $n$, such that the trial with the shortest MIT was ranked first and the trial with the longest MIT was ranked $n$th (where $n$ is the number of trials in that design cell). We then fitted a polynomial regression model to the $\mathrm{x}$-velocity profiles that included MIT rank as the only covariate and polynomial terms up to the 15 th order. Terms that did not explain significant variance were dropped from the model so that only significant coefficients were used to generate predicted $\mathrm{x}$-velocity profiles (one for each trial). The specific OPTA procedure used here has been described elsewhere and we point the interested reader to those publications for a detailed description (cf., Finkbeiner et al., 2014; Quek \& Finkbeiner, 2014). Following the OPTA procedure, we averaged the fitted $x$-velocity values from the first $150 \mathrm{~ms}$ of the reaching movement and submitted this mean value, which we refer to as initial $x$-velocity, to a linear mixed-effects model (LMM, Bates, 2005), with MIT semi-decile included as a fixed effect.

To visualize the effect of target-viewing time (i.e., MIT latency) on the complete reaching response (see the upper panel of Fig. 3 for the distribution of MIT latencies in Experiment 1), we used the corresponding MIT rank values to group the $\mathrm{x}$-velocity profiles into 20 bins of equal proportion (i.e., semi-deciles). The mean trajectory (averaged across subjects) from each inter-decile range of the MIT ranks is presented in the bottom panel of Fig. 3 for the incongruent condition. It shows that the longer subjects delay their movement, the more quickly they reach peak positive $\mathrm{x}$-velocity (i.e., movement in the correct direction). The same was true for congruent and neutral conditions.

\section{Results}

The final (i.e., movement end-point) accuracy of subjects' reaching responses was very high, $99.6 \%$ overall, $99.9 \%$ in the congruent condition, and $99.2 \%$ in the incongruent condition. Presumably this was because even if the initial movement is in the wrong direction, subjects take approximately 1 second to finish their movement, which appears to be sufficient time to correct any initial waywardness in the hand's flight path and arrive at the correct location. Despite the very high overall accuracy, a paired t-test indicated a reliable difference between the congruent and incongruent conditions $(t(17)=2.66, p=0.016)$. The accuracy in the neutral condition $(99.7 \%)$ was reliably different from the incongruent condition $(t(17)=2.36, p=0.03)$, but the difference between the congruent and neutral conditions was not reliable $(t(17)<2)$.

The analyses of initial $\mathrm{x}$-velocity was limited to correct responses. We used an incremental model comparison approach in which we evaluated the contribution of each term by comparing a model that included the term of interest with a model that did not include that term. In each case, likelihood tests were used to indicate which model should be preferred. Only terms that significantly improved the fit of the model were included. We included subjects as a random effect and Trial Type (congruent, neutral, incongruent) and MIT percentile (semi-decile) as fixed effects. Given the curvilinear relationship between initial x-velocity and MIT, we included up to fourth order terms for MIT percentile in the model (including higher-order terms did not improve the fit of the model further).

We report the coefficients $(b)$, standard errors $(S E)$, and $t$ values for the model that provided the best fit to the data. As is typical in reporting the results of LMM analyses, we take a coefficient magnitude of at least twice its standard error (i.e. $|t|$ $>2$ ) as our criterion for significance (cf., Kliegl, Masson, \& Richter, 2010; Kliegl, Wei, Dambacher, Yan, \& Zhou, 2010). For the purposes of exposition we only report terms that are linear in MIT percentile, but note that including the higherorder terms leads to the same interpretations. Coefficients for the trial-type factor used the congruent condition as a baseline, so that negative coefficients indicate smaller $\mathrm{x}$-velocities relative to the congruent condition.

There was a strong effect trial type with a larger $\mathrm{x}$ velocity for the congruent condition than the incongruent $(b=-0.58, \mathrm{SE}=0.01, t=52.48)$ and neutral $(b=-0.30$, $\mathrm{SE}=0.01, t=28.67)$ conditions. There was also a significant increase in $\mathrm{x}$-velocity with MIT percentile $(b=$ 42.67, $\mathrm{SE}=0.68, t=61.94)$ and an interaction between trial type and MIT percentile (incongruent: $b=-15.03$, SE $=0.98, t=15.27$; neutral: MIT $b=-6.93, \mathrm{SE}=0.96, t=$ 7.20). The nature of this interaction is clear in Fig. 4. Movements initiated within the first $\sim 100 \mathrm{~ms}$ after target onset were, on average, "down the middle." That is, the net movement was neither in the correct (positive $\mathrm{x}$ velocities) nor in the incorrect (negative $\mathrm{x}$-velocities) direction. In contrast, movements that commenced after $\sim 100 \mathrm{~ms}$ were dramatically different depending on Trial Type. On congruent trials, initial $\mathrm{x}$-velocity steadily increased with target viewing time; on incongruent trials, initial $\mathrm{x}$-velocity became increasingly negative (i.e., in the wrong direction) for a period of time $(\sim 150 \mathrm{~ms})$ before becoming positive again. On neutral trials, the net movement was "down the middle" (x-velocities were not 


\section{Grouping Trajectories by Target Viewing Time}
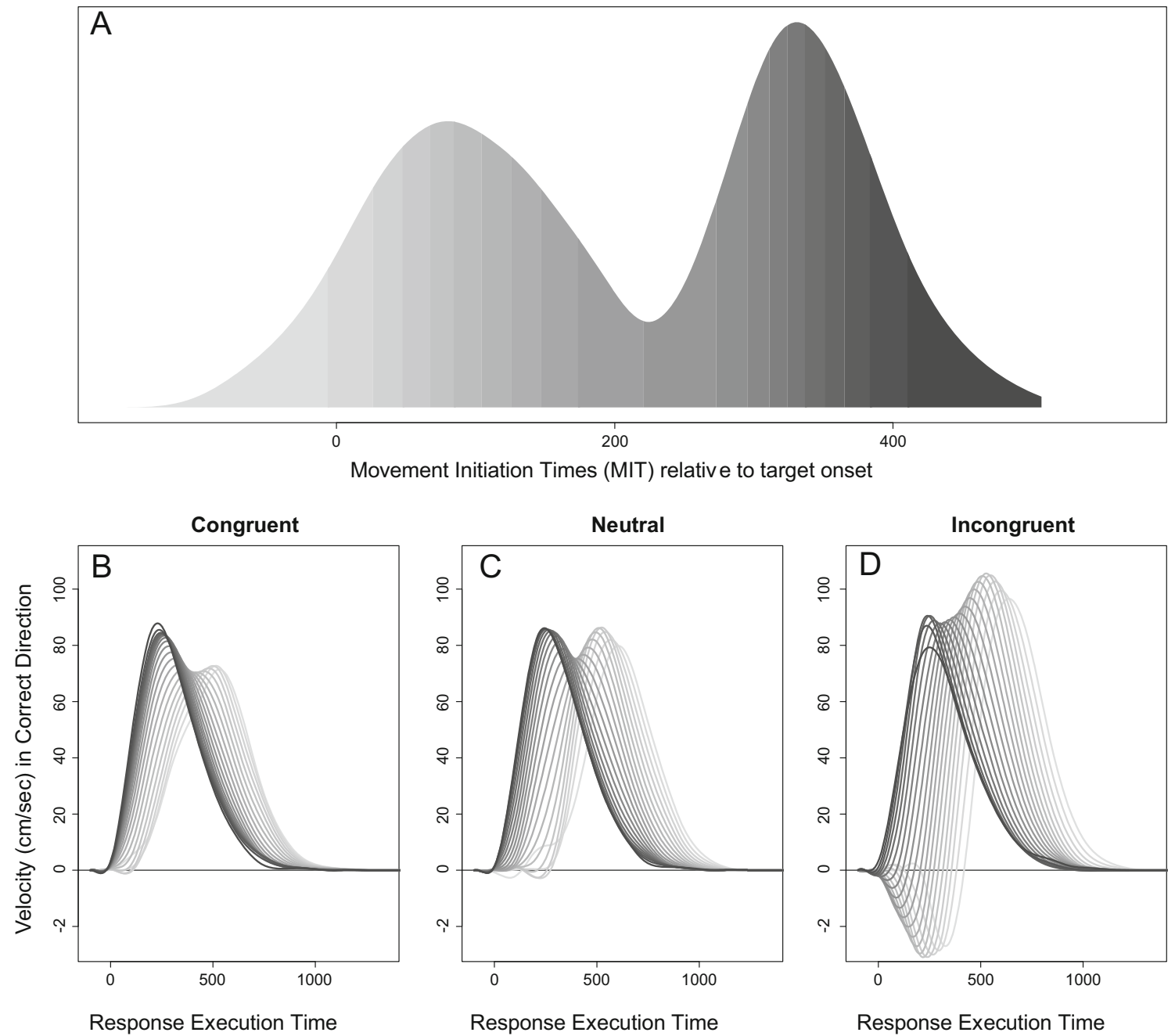

Fig. 3 Distribution of movement initiation times (MITs) (a) and corresponding mean $\mathrm{x}$-velocity profiles (b) from Experiment 1 . The lightest gray color corresponds to the trials with the earliest MITs (first semidecile) and the darkest gray color corresponds to the longest MITs (20th semi-decile). The horizontal line represents an x-velocity of zero; values above this line represent a net movement in the correct direction and values below this line indicate a net movement in the incorrect direction different from zero) for movements that were initiated within the first $\sim 300 \mathrm{~ms}$ after target onset. ${ }^{3}$

To better understand the unfolding of the effect of trial type, we then conducted a series of one-sample t-tests (Bonferroni corrected, 17 degrees of freedom) to determine when (i.e., at which MIT percentile) the net movements were significantly different from zero. On congruent trials, the initial $x$-velocities were all reliably greater than zero (net movement was in the correct direction) beginning with movements that commenced in the seventh MIT percentile ( $109 \mathrm{~ms})$. On incongruent

\footnotetext{
${ }^{3}$ We note that the position of the neutral stimulus (upper vs. lower hemifield) did not have a reliable effect on responses. We also note that the un-averaged $\mathrm{x}$-velocities from the neutral condition (one for the upper stimulus position and one for the lower position) both overlap with the aggregated values depicted for the neutral condition in Fig. 4.
}

trials, the pattern was biphasic. First, the initial x-velocities were reliably less than zero (net movement was in the wrong direction) for movements that commenced in the eighth through the 12th MIT percentile $(\sim 128 \mathrm{~ms}$ through $\sim 287 \mathrm{~ms}$ ). They then became reliably greater than zero for movements that commenced in the 17th through the 20th MIT percentile ( $\sim 356 \mathrm{~ms}$ through $\sim 430 \mathrm{~ms})$. On neutral trials, initial $\mathrm{x}$-velocities were reliably greater than zero beginning with movements that commenced in the 13th MIT percentile ( $\sim 300 \mathrm{~ms})$.

\section{Discussion}

Experiment 1 revealed that the initial direction of movements that commence $\sim 150 \mathrm{~ms}$ after target onset in both 
Initial $\mathrm{x}$-velocity by Trial Type in Experiment 1

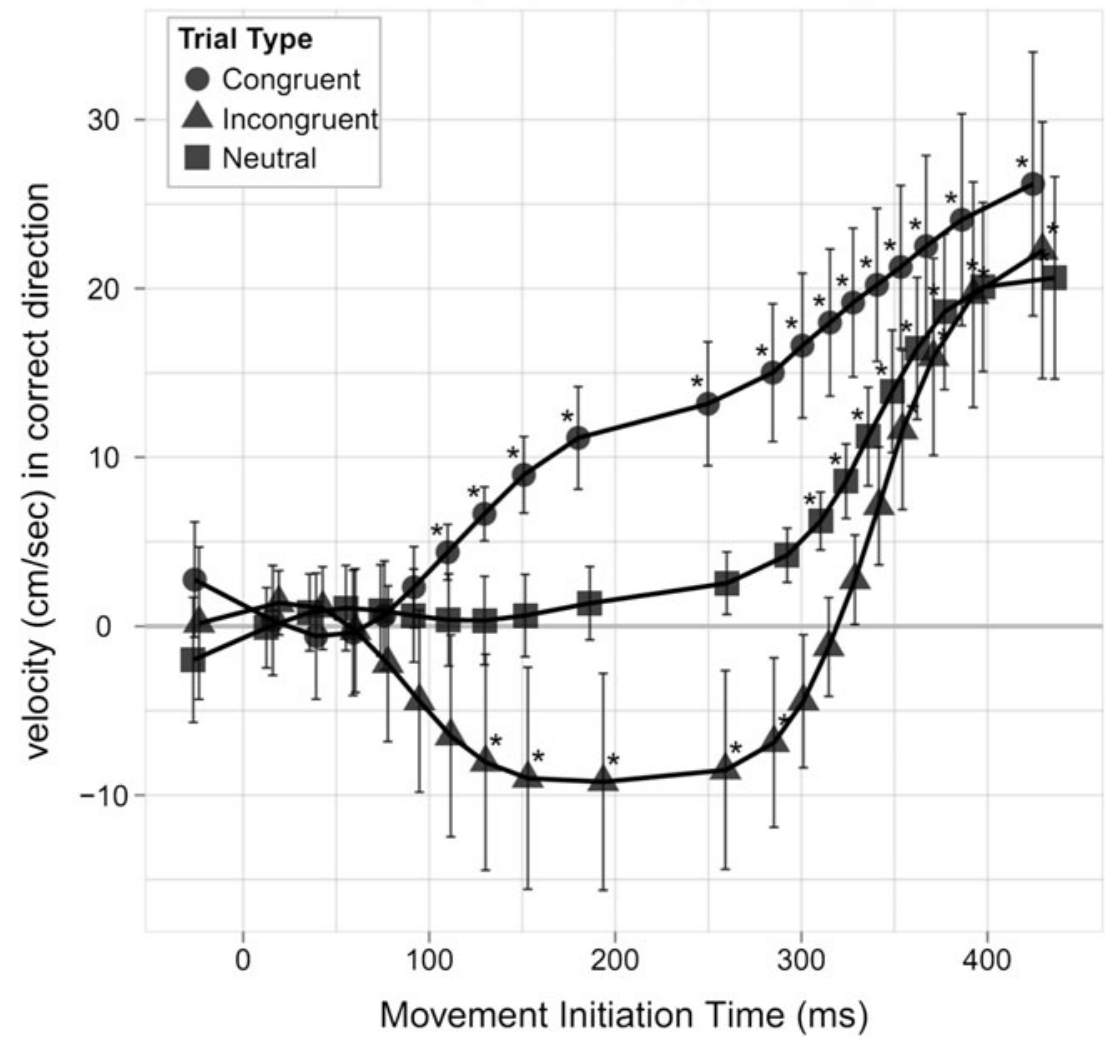

Fig. 4 Initial x-velocity by movement initiation time and Trial Type in Experiment 1. Error bars represent within-subjects $95 \%$ confidence intervals and asterisks indicate $\mathrm{x}$-velocities that are reliably different from zero (Bonferroni corrected)

the congruent and incongruent conditions is strongly influenced by the location of the stimulus. This clear influence of the task-irrelevant spatial information stands in sharp contrast to the lack of any influence by the taskrelevant information during this early phase of stimulus processing in the neutral condition (where the stimulus' position is orthogonal to the response). However, the influence of stimulus location on the initial direction of the reaching response is short-lived. The initial direction of movements that commence just $150 \mathrm{~ms}$ later $(\sim 300 \mathrm{~ms}$ after target onset) is clearly controlled by the meaning of the target word, not its location. This is seen most clearly in the comparison between the incongruent condition and the neutral condition, where the target meaning should be the only influence on $\mathrm{x}$-velocity. This finding appears to clearly reject a pure magnitude-difference explanation, which assumes that both task-relevant and taskirrelevant information are available at the same time.

However, it is possible that encoding of task-relevant information was slowed in the neutral condition because participants paid less attention to the stimulus locations above and below fixation than to the locations to the left and right of fixation. This may have occurred because, even though the overall proportion of neutral trials was the same as the proportion of congruent and incongruent trials (one-third each), the proportion of stimuli in the upper and lower locations was less than it was in the left and right locations (one-sixth vs. one-third). Experiment 2 addresses this potential confound by using each of the four possible stimulus locations equally often, so that neutral trials were twice as common as congruent and incongruent trials.

\section{Experiment 2}

An additional motivation for Experiment 2 was to establish the generality of the pattern of findings observed in Experiment 1 by using red and green squares (as opposed to words) as targets. This allows us to compare the dynamics of the Simon effect that we have observed with targets that enjoy over-learned response mappings (i.e., the words "LEFT" and "RIGHT" in Experiment 1) with the Simon effect that arises with targets that share an arbitrary relationship with response location (i.e., the colors red and green mapping onto "left" and "right" responses). We also implemented an improved SOA manipulation in order to eliminate the bimodal distribution 
of MITs shown in Fig. 3 and to obtain a more even spread concentrated around the critical middle region of the MIT range.

\section{Method}

\section{Subjects}

Eighteen undergraduates (ten female) were recruited from the same subject pool as Experiment 1. Subjects participated for course credit and all had normal or corrected-to-normal vision and all reported being right-handed.

\section{Apparatus}

The same apparatus was used in Experiments 1 and 2 with the only difference being that we added the appropriately colored squares to the response panels in Experiment 2 (see Fig. 2).

\section{Design and procedure}

The procedure was identical to that of Experiment 1 with some small exceptions. In particular the two targets were crossed with the four different stimulus locations (right, top, left, bottom) to yield eight different target-by-location combinations. As mentioned, this design change led to $25 \%$ of the trials being congruent, $25 \%$ being incongruent, and $50 \%$ being neutral, with $25 \%$ of the neutral stimuli being presented above fixation and $25 \%$ below. The eight different trial combinations were then rotated through each target-to-go-signal SOA for a total of 40 fully-crossed trials per block. The SOAs were arranged to yield overlapping response windows, with a $40 \%$ probability of a 0 -ms SOA (target and go signal appeared simultaneously), a $40 \%$ probability of a $150-\mathrm{ms}$ SOA (target appeared $150 \mathrm{~ms}$ before the go signal), and a $20 \%$ probability of a $250-\mathrm{ms}$ SOA. The mapping between target color and response side was counterbalanced across subjects so that half of the subjects reached to the left for red colors and to the right for green colors. This mapping was reversed for the remaining subjects. The experiment began with two blocks of practice trials $(\mathrm{N}=80)$, followed by eight blocks of experimental trials $(\mathrm{N}=320)$.

\section{Results}

The final accuracy of subjects' reaching responses was again very high, with an overall accuracy rate of $99.9 \%$ : $100 \%$ in the congruent, $99.8 \%$ in the incongruent, and $99.9 \%$ in the congruent conditions. None of these differences were reliable. Figure 5 shows MIT distribution for Experiment 2. As in Experiment 1 it spread over a range from before the target onset to around $400 \mathrm{~ms}$ after, but due to the use of SOAs with overlapping response windows (compared to SOAs with non- overlapping response windows in Experiment 1) the MIT distribution is more evenly distributed over the range in Experiment 2. The bottom panel of Fig. 5 presents the mean trajectory for each semi-decile for the congruent condition of Experiment. Just as in Experiment 1, it shows that the longer subjects delay their movement, the more quickly they reach peak positive $x$-velocity, and again as in Experiment 1 the same was true in the incongruent and neutral conditions.

The same incremental model comparison approach was used as in Experiment 1, except that we only included up to a third of the order terms for MIT percentile (including higherorder terms did not improve the fit of the model further). Again, in the interest of brief exposition, we only report the linear terms from the final model. There were strong effects of trial type (incongruent: $b=-0.80, \mathrm{SE}=0.01, t=60.48$; neutral: $b=-0.33, \mathrm{SE}=0.01, t=28.88)$, MIT percentile $(b$ $=32.67, \mathrm{SE}=0.69, t=47.45$ ), and their interaction (incongruent: $b=-25.03, \mathrm{SE}=0.96, t=26.14$; neutral: $b=-10.04$, $\mathrm{SE}=0.83, t=11.98$ ). Figure 6 illustrates the nature of the interaction, which was very similar to Experiment 1. All movements that were initiated within the first $\sim 100 \mathrm{~ms}$ of target onset were, on average, "down the middle" where the net movements were neither in the correct nor incorrect direction, but movements that commenced after $\sim 100 \mathrm{~ms}$ were markedly different depending on trial type. On congruent trials initial $x$-velocity steadily increased with target viewing time; on incongruent trials, initial x-velocity became increasingly negative for a period of time $(\sim 150 \mathrm{~ms})$ before becoming positive again. On neutral trials, $\mathrm{x}$-velocities were not different from zero for movements that were initiated within the first $\sim 250 \mathrm{~ms}$ of target onset.

We again conducted a series of one-sample t-tests (Bonferroni corrected, 17 degrees of freedom) to determine at which MIT percentile the net movements were different from zero. On congruent trials, the initial x-velocities were reliably greater than zero (in the correct direction) beginning with movements that commenced in the seventh ${ }^{\mathrm{h}}$ MIT percentile ( $129 \mathrm{~ms})$. On incongruent trials, the pattern was again biphasic; first, the initial x-velocities were reliably less than zero (in the wrong direction) for movements that commenced in the eighth through the 14th MIT percentile $(\sim 147 \mathrm{~ms}$ through $\sim 232 \mathrm{~ms}$ ), whereas from the 19th MIT percentile $(\sim 311 \mathrm{~ms}$ through $\sim 352 \mathrm{~ms}$ ) they were reliably greater than zero. On neutral trials, initial x-velocities were reliably greater than zero beginning with movements that commenced in the 12th MIT percentile ( 206 ms).

\section{Discussion}

Experiment 2 largely replicated the pattern of differences seen in Experiment 1. The absolute values of effects tended to be faster than in Experiment 1, which may be attributable to the shorter average SOA in Experiment 2 causing participants to 


\section{Grouping Trajectories by Target Viewing Time}

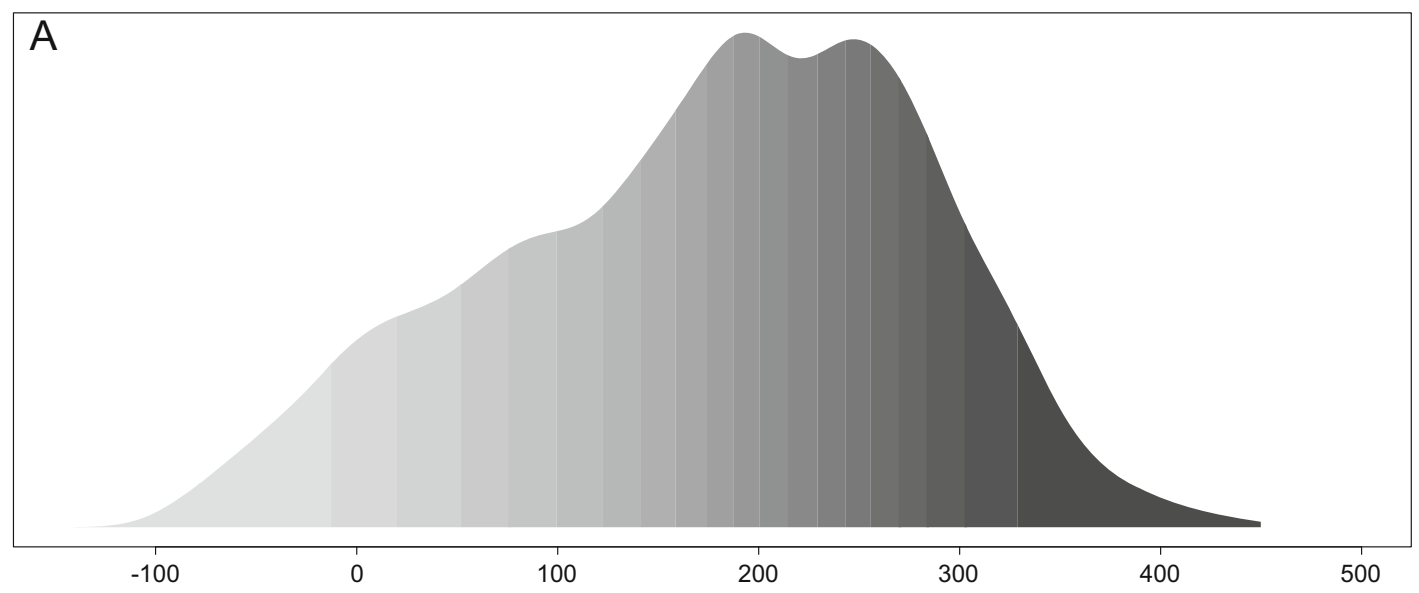

Movement Initiation Times (MIT) relative to target onset
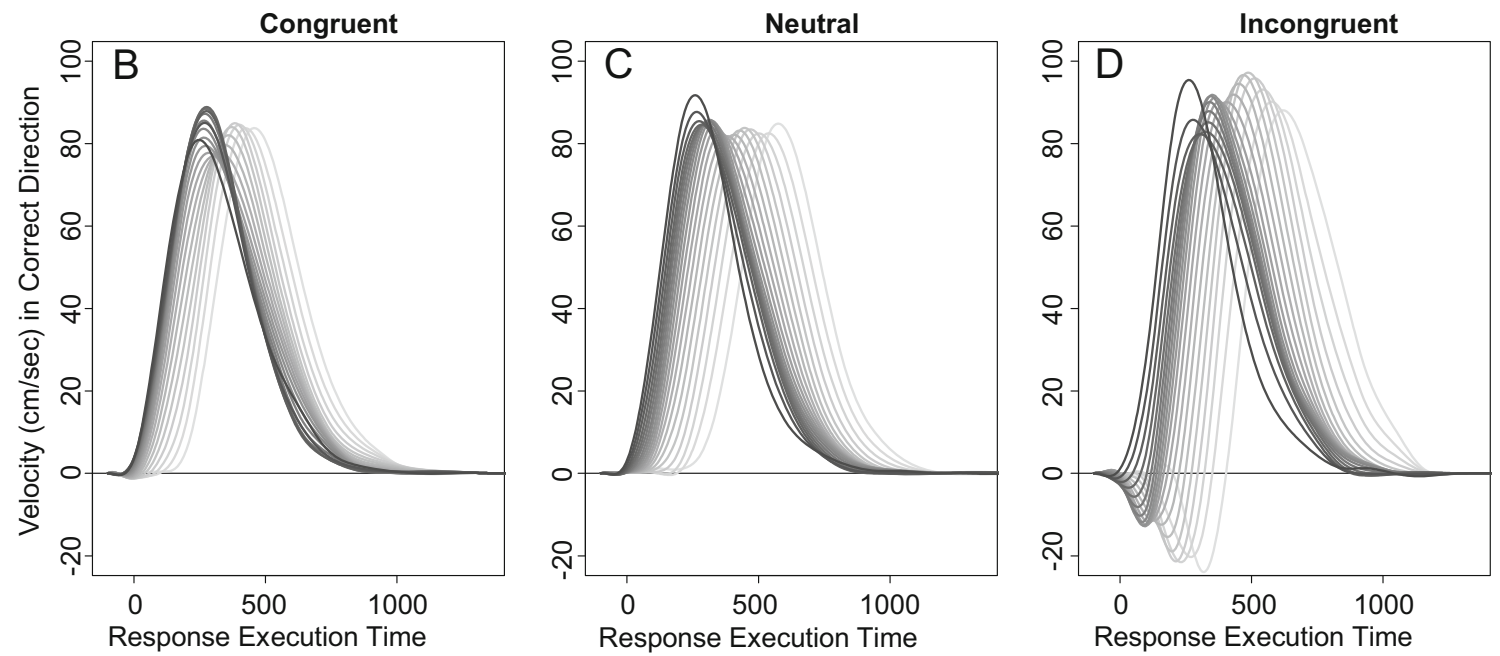

Fig. 5 Distribution of movement initiation times (MITs) (a) and mean xvelocity profiles (b) from Experiment 2 . The lightest gray color corresponds to the trials with the earliest MITs (first semi-decile) and the darkest gray corresponds to the longest MITs (20th semi-decile). The horizontal line represents an $\mathrm{x}$-velocity of zero speed their overall rate of processing. However, once again, the initial direction of movements that commenced $\sim 100 \mathrm{~ms}$ after target onset was governed by the location of the stimulus, but this influence was short-lived, with the initial direction of movements that commence just $200 \mathrm{~ms}$ later $(\sim 300 \mathrm{~ms}$ after target onset) controlled by the meaning of the target. Similarly, the neutral condition indicated that task-relevant information became available approximately $75 \mathrm{~ms}$ after the irrelevant spatial information. In contrast to Experiment 1, the slower onset of the neutral conditions cannot be explained by participants paying attention to locations in proportion to the probability that they will contain a stimulus, as the locations used in the neutral condition had the same probability as the locations used in the congruent and incongruent condition.

Overall, the results of Experiments 1 and 2 strongly reject the pure magnitude-difference explanation of the Simon effect depicted in Fig. 1. These results confirm an assumption about the time course of processing that is common to many theories of the Simon effect, that irrelevant spatial information becomes available earlier than task-relevant information. The depth of the implications of these results depend somewhat on the theoretical framework from which they are viewed. For example, they are difficult to accommodate if it is assumed that relevant characteristics have been isolated and stimulusstimulus conflicts are resolved in stages completed prior to the activation of response representations. In contrast, a time difference could easily be added to a model using Cohen et al.'s (1990) PDP framework even though it originally assumed only a magnitude difference. In either case, it is important to note that our results do not rule out some role for differences in magnitude modulating the size of the Simon effect, a point that we now elaborate. 


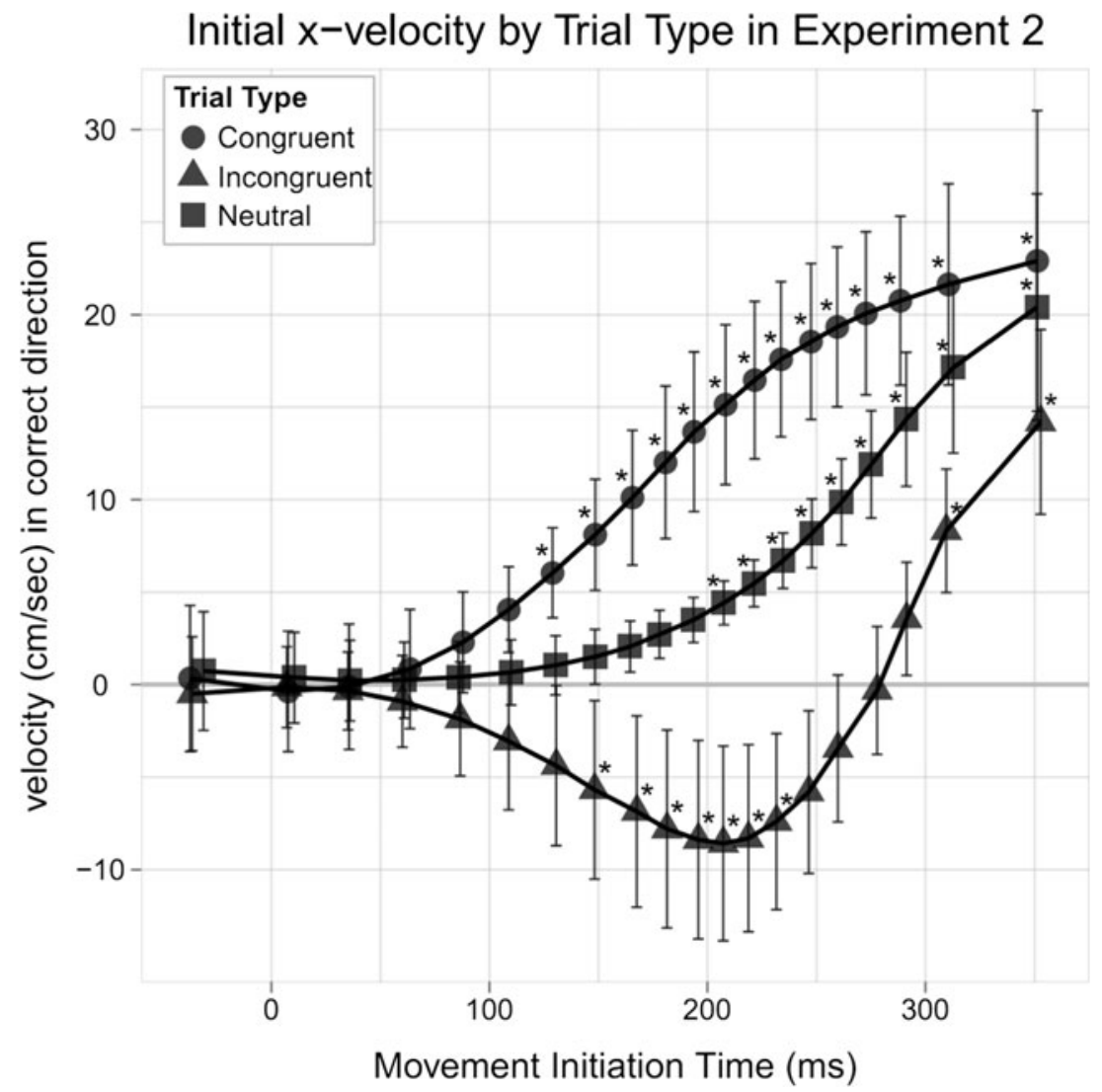

Fig. 6 Initial x-velocity by movement initiation time and Trial Type in Experiment 2. Error bars represent within-subjects $95 \%$ confidence intervals and asterisks indicate $\mathrm{x}$-velocities that are reliably different from zero (Bonferroni corrected)

In the next section we examine sequential effects, which have not, to our knowledge, been investigated before with the reach-to-touch paradigm. Sequential effects have been observed in other paradigms previously, whereby the magnitude of the Simon interference effect on the current trial varies depending on whether the previous trial was congruent or incongruent. Because these effects are relatively small, we were required to combine the data from Experiments 1 and 2 in order to have sufficient power to detect them. We first review the literature pertaining to these sequential effects in the button-press paradigm, and then report the results of our reach-to-touch analysis.

\section{Sequential effects}

Sequential effects in the Simon task were first reported in a pair of conference abstracts (Mordkoff, 1998, and Stürmer, Leuthold, \& Sommer, 1998, as cited in Hommel, Proctor, \& Vu, 2004; see also Stürmer et al., 2002), and have been subsequently replicated by other researchers. Interference (i.e., RT for incongruent minus congruent trials) was strong after a congruent trial but reduced or absent on trials following an incongruent trial. In both cases an explanation was proposed in terms of the dual-route model, whereby the type of the previous trial controlled the influence of the direct route. The two explanations differed in that Stürmer et al. (2002) proposed that inhibitory control was only exerted after incongruent trials, whereas Mordkoff (1998) allowed for an increase in the effect of the direct route, and so facilitated responding after congruent trials.

Both explanations are similar to the conflict resolution and cognitive control theories of the Stroop effect (Botvinick, et al., 2001; Miller \& Cohen, 2001), making the observed sequential changes an integral part of the Simon effect. Hommel et al. (2004) put forward a different view, proposing that they were a separate phenomenon related to the automatic consequences of storing associations between stimulus locations and response locations ("event files") on each trial (e.g., Hommel, Müsseler, Aschersleben, \& Prinz, 2001). Hommel et al. (2004) pointed out that associations made on an incongruent trial either completely match or completely mismatch associations on a following incongruent trial, and so should either facilitate or not affect current performance. In contrast, the associations will always contain one element that matches a subsequent congruent trial and one that mismatches, creating a conflict that could slow responding. As a consequence the magnitude of the interference effect is reduced. The opposite 
occurs following a congruent trial, so that the magnitude of the interference effect is increased. That is, the effect of event files is completely confounded with the sequential effects reported by Mordkoff (1998) and Stürmer et al. (1998), and so could provide an alternative explanation not requiring cognitivecontrol mechanisms.

Although sequential effects are a robust finding with button-press responding, they have not been previously investigated in the reach-to-touch paradigm. Given the differences between these paradigms it is possible that they may be attenuated or absent in our experiments. First, each trial takes much longer than in the button-press paradigm, due to the extended response process, so this may weaken effects from one trial to another. Second, errors are rare or non-existent in the reach-totouch paradigm, in the sense that the final location that participants touch is almost always correct, which may lessen the effects of conflict. However, conflict clearly does occur earlier in the trial, as indicated by the initial velocities in the incorrect direction shown in Figs. 4 and 6 for incongruent trials, so it is possible that this will trigger control processes that will create sequential effects. Similarly, event-file effects could create sequential effects as both stimulus and response locations exist in the reach-to-touch paradigm just as they do in the buttonpress paradigm. However, if the larger temporal gap between the initial processing of the stimulus and the final response weakens their stored association it is possible that event-file related sequential effects would be attenuated.

\section{Results}

We combined the data from Experiments 1 and 2 and submitted it to the same analyses described above, but we now included Previous Trial Type as a factor. By including this additional factor, we were restricted in our distribution analysis to binning the data into ten groups of equal proportion (deciles) instead of the semi-deciles that we used above (see Fig. 7; note that for clarity we omit error bars in this plot). Our model comparison procedure revealed that including Previous Trial Type improved the model's fit to the data $\left(\chi^{2}(2)=39.9\right.$, $\mathrm{p}<.001$ ). Including Experiment did not improve the fit of the model but, importantly, including the three-way interaction between Current Trial Type, Previous Trial Type, and MIT Quantile did $\left(\chi^{2}(12)=110.5, p<.001\right)$. Thus the final model that provided the best fit to the data included the fixed effects Current Trial Type, Previous Trial Type, and MIT Quantile, along with Subjects as a random effect.

Given the complexity of the final model, only a few representative linear terms from that model are reported here, which are Current Trial Type (Incongruent current $: b=-0.17, \mathrm{SE}=$ $0.004, t=38.07$; Neutral $\mathrm{current}_{\mathrm{i}}: b=-0.08, \mathrm{SE}=0.004, t=$ 20.58), Previous Trial Type (Incongruent prev $: b=-0.02$, SE $=0.004, t=5.7$; Neutral $\mathrm{p}_{\text {rev }}: b=0.004, \mathrm{SE}=0.004, t=.98$ ), and MIT percentile $(b=9.1, \mathrm{SE}=0.51, t=18.74)$. All of the interaction terms between Current and Previous Trial Types were reliable (each $|t|>2$ ). With respect to the three-way interaction between Current Trial Type, Previous Trial Type, and MIT Quantile, this appears to have been carried primarily by the incongruent trials (Incongruent ${ }_{\text {prev }} \times$ Incongruent $_{\text {current }} \times$ MIT Quantile $\left._{\text {linear: }}: b=4.8, \mathrm{SE}=0.72, t=6.7\right)$, with all the other terms only marginally significant. The nature of the three-way interaction is clear in Fig. 7, where we see (1) that the effect of Previous Trial Type is strongest when the current trials are incongruent and (2) that participants are most affected by the position of the incongruent stimulus on the current trial when the previous trial was congruent.

To better understand how Previous Trial Type affected the relationship between Current Trial Type and MIT Quantile (i.e., the time course of the Simon effect on the current trial), we conducted a series of one-sample t-tests (Bonferroni corrected). Although the largest sequential differences occurred when the current trial was incongruent there was some evidence of a difference on congruent trials for fast MITs. On $\mathrm{CC}$ trials $\left(\right.$ Congruent $_{\text {previous }}$, Congruent current $_{\text {) }}$, the initial $\mathrm{x}$ velocities were reliably greater than zero (net movement was in the correct direction) beginning with movements that commenced in the fourth MIT percentile ( 129 ms from stimulus onset). On IC trials (Incongruent previous $_{\text {, Congruent }}$ current $_{\text {) }}$, the initial $\mathrm{x}$-velocities were reliably greater than zero beginning with the fifth MIT percentile $(\sim 173 \mathrm{~ms})$. It should be noted that the use of one-sample t-tests may be too anticonservative in this case and that the initial $\mathrm{x}$-velocities for $\mathrm{CC}$ and IC trials were not different from one another in the fourth MIT percentile. Nevertheless, given the within-subjects and within-items design, it seems informative that, if the preceding trial was incongruent, our subjects needed to view a congruent target stimulus for an additional $\sim 44 \mathrm{~ms}$ before the directional velocity of the initial segment of their movement was reliably greater than zero.

For CI trials (Congruent previous $_{\text {, Incongruent }}$ current ), the pattern was biphasic. First, the initial $\mathrm{x}$-velocities were reliably less than zero (net movement was in the wrong direction) for movements that commenced in the fourth through the seventh MIT percentiles $(\sim 129-267 \mathrm{~ms})$ and then they were reliably greater than zero again for movements that commenced in the tenth MIT percentile $(\sim 368 \mathrm{~ms})$. For II trials (Incongruent $_{\text {previous }}$, Incongruent current $_{\text {) }}$, the initial $x$ velocities were reliably less than zero for a shorter period of time (fifth and sixth MIT percentiles) compared to CI trials, and were reliably greater than zero for a longer period of time (ninth and tenth MIT percentiles). To look at the differences between CI and II trials more closely, we analyzed the critical subset of MIT percentiles (four through seven) to see if there was an interaction between trial type (CI and II) and MIT percentile (four through seven). This follow-up analysis revealed a reliable interaction $(b=.61, \mathrm{SE}=0.15, t=4.3)$. The nature of this interaction (see Fig. 7) suggests that, when 


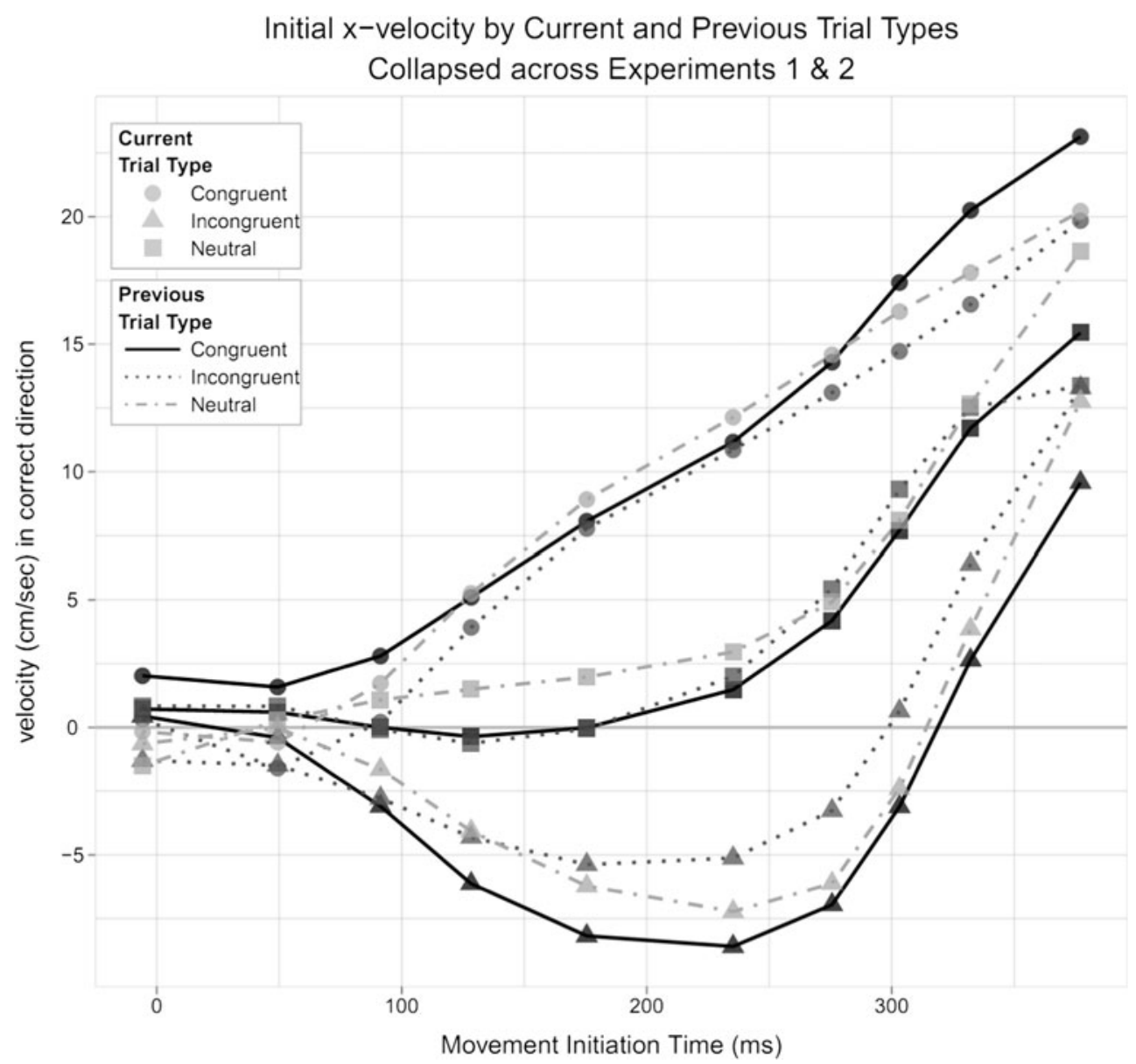

Fig. 7 Initial x-velocity by movement initiation time, Current Trial Type, and Previous Trial Type

presented with an incongruent target, our subjects' initial movements were "captured" by the irrelevant position of the target stimulus earlier ( $\sim 44 \mathrm{~ms})$ and for a longer period of time ( $\sim 45 \mathrm{~ms}$ ) if the preceding trial had been congruent.

\section{Discussion}

Taken together, our results suggest that sequential effects are observed in the reach-to-touch paradigm, but that they are perhaps lesser than in the button-press paradigm. For example, in their first experiment Stürmer et al. (2002) reported an average interference effect of $66 \mathrm{~ms}$ after congruent trials and $0 \mathrm{~ms}$ after incongruent trials when there were equal numbers of each trial type. In contrast, Fig. 7 shows that after a congruent trial there was still a substantial movement in the wrong direction on incongruent trials. One possibility is that our inclusion of a neutral condition, and hence decreased percentage of incongruent trials, might decrease the need to exert control to manage conflict, thus reducing sequential effects. Although not directly analogous (as they did not include neutral trials) Stürmer et al. also reported substantial sequential differences in interference effects when they manipulated the percentage of incongruent trials ( $80 \%$ : $56 \mathrm{~ms}$ after congruent and $-38 \mathrm{~ms}$ after incongruent; $20 \%$ : $101 \mathrm{~ms}$ after congruent and $-2 \mathrm{~ms}$ after incongruent). Hence, it seems likely that there are other causes of the attenuated sequential effects in our experiments, such as slower response production and/or lower error rates.

Although attenuated, there were clear sequential effects, manifesting mostly on incongruent trials. In this case there appeared to be a decrease in the overall magnitude of interference from the irrelevant information after an incongruent trial. That is, $x$-velocity became significantly negative (i.e., in the wrong direction) later and changed back to positive more quickly in an incongruent trial that followed another incongruent trial. This magnitude effect is consistent with Hommel et al.'s (2004) event-file explanation, which might be expected to have a largely additive effect across the time course of processing. It is less consistent with Ridderinkhof's (2002a) interference-suppression explanation to the degree that this control mechanism is supposed to come into play later in a trial and so might be expected to predict stronger differences given stronger control is exerted after an incongruent trial - for longer MITs. However, it is possible that sequential effects are caused by more than one mechanism, explaining the short MIT differences, and that interference-suppression effects do 
not become evident until beyond the range of MITs that we examined.

Although sequential effects manifested predominantly on incongruent trials there is some suggestion in our data that, early in the time course, a previous congruent trial facilitates processing relative to a previous incongruent trial. This was suggested by $\mathrm{x}$-velocity trials becoming significantly positive earlier on congruent trials when they followed another congruent trial than when they followed an incongruent trial. This pattern is consistent with control processes increasing the influence of the direct spatial route following a trial on which it helps responding. It is also consistent with an identity repetition benefit (i.e., when the two congruent trials are identical) as proposed by Hommel et al.'s (2004) theory. Again the possibility of multiple causes means that further research will be required to disentangle these possibilities.

In summary, the results of our sequential analysis demonstrate both that evidence for the magnitude-based account can be detected by our response-signal reach-to-touch methodology, and that this account does have a role in the Simon paradigm. In our data that role appears to be dissociated from the main time-based effect, appearing as a secondary and smaller modulation of it. However, it is possible that with a shorter gap between trials and with different proportions of congruent and incongruent trials the magnitude-based effect may have a larger effect.

\section{General discussion}

The present study establishes several findings with important implications for the literature on choice conflict. First, by coupling the reach-to-touch paradigm with the response signal procedure in the Simon task we were able to elicit responses across a wide range of stimulus-viewing times. This allowed us to establish the minimum stimulus-viewing duration needed by subjects to produce "reliable movements" - that is, movements that were either reliably correct or reliably incorrect (i.e., not guesses). In both Experiment 1 and Experiment 2, the minimum stimulus-viewing duration to produce reliable movements was longer in the neutral condition than it was in both the congruent and incongruent conditions. This finding is consistent with the time-difference account, which holds that activation from task-irrelevant spatial attributes becomes available earlier than activation from task-relevant attributes. Importantly, this finding is inconsistent with the magnitudedifference account, which holds that activation from both relevant and irrelevant attributes become available at the same time, but to different degrees.

Second, not only was the minimum stimulus-viewing duration for reliable movements less in the congruent and incongruent conditions, but the first reliable movements in these conditions were in the correct direction for congruent trials and in the incorrect direction for incongruent trials. The finding that reliable movements were produced with shorter stimulus-viewing times in the congruent and incongruent conditions (relative to neutral), and that the first reliable movements in these conditions were in the direction of the stimulus, provides strong support for the time-difference account of the Simon effect.

\section{The slow neutral condition}

Although most behavioral studies of the Simon effect do not include a neutral condition, the typical finding with those that do is that the RTs in the neutral condition are slower than the RTs in the congruent condition and faster than the RTs in the incongruent condition (Hommel, 1993b; Simon \& Acosta, 1982; Simon \& Craft, 1970). This standard pattern of results is frequently taken as support for the notion that interference arises as a function of the temporal overlap in response activation attributed to the transient encoding of spatial information and response activation attributed to the slower, but more persistent, encoding of the task-relevant information. When the two sources of information conflict, as they do in the incongruent condition, it takes longer to generate a response because the conflict must first be resolved. On this standard interpretation of the Simon effect, responses in the incongruent condition will necessarily lag behind those in the neutral condition because there is no conflict to resolve in the neutral condition.

However, we found in our study that the longest minimum stimulus-viewing time was in the neutral condition. How should our finding be reconciled with the standard dualroute account of the Simon effect? In our paradigm, subjects were given ample time to complete their movements and so they quickly learned that they could make a correct response even when commencing their reaching movement within a few milliseconds of stimulus onset. As a result, subjects could initiate their reaching responses with impunity. The virtue of this paradigm is that it relieves the subject of having to resolve conflict prior to generating a response, and, in turn, provides a window into the relative strengths of competing sources of information at different points in stimulus processing. With this in mind, our finding, where the longest minimum stimulus-viewing time occurred in the neutral condition, is consistent with the standard dual-route account of the Simon effect. Reliable movements could be generated in the congruent and incongruent condition with shorter stimulus-viewing times because, at the point when the earliest movements were initiated, the rapidly encoded spatial information was sufficient to produce reliable responses in the direction of the stimulus. In contrast, because our neutral stimuli were presented along the vertical meridian, the spatial information encoded by the fast, direct route would have been orthogonal to the response. Thus, the only way to generate a reliable response in 
the neutral condition would have been via the encoding of the task-relevant attributes of the stimulus, which is posited to be in the province of the slower, cognitive route. It follows from the dual-route account of the Simon effect then, that in our paradigm, in which subjects are not required to resolve conflict prior to initiating a response, the neutral condition should yield the longest minimum stimulus-viewing times.

Importantly, the minimum stimulus-viewing times in the neutral condition can be used to index the lower bound on the time needed for the 'cognitive' route to encode the taskrelevant attribute of the stimulus and gain control of the response formulation process. The finding that the minimum stimulus-viewing time was longest in the neutral condition is very important for our present purposes because it allows us to adjudicate between the pure time-difference and magnitudedifference accounts. As we indicated in the Introduction (see also Fig. 1), both of these explanations provide a straightforward account of the time course of the Simon effect with endpoint measures, where the magnitude is typically the largest in early responses and smaller in later responses (but see Introduction for a review of exceptions to this general pattern). The critical distinction between the time- and magnitudedifference accounts is in when the task-relevant information becomes available. According to the time-difference account, the task-relevant information becomes available after the taskirrelevant information. The magnitude-difference account, on the other hand, posits that both task-relevant and taskirrelevant information becomes available at similar times in stimulus processing, but that the magnitude of activation produced by the task-irrelevant information is greater for a brief amount of time early on in stimulus processing. Following from this latter account, the minimum stimulus-viewing times should be roughly equal across the congruent, incongruent and neutral conditions, with the only difference being in the magnitude of subjects' initial velocities. Our findings are clearly inconsistent with this prediction of the magnitudedifference account.

One might note at this point that our rejection of the magnitude-difference account depends on the lack of a positive result in the neutral condition juxtaposed with positive results in the congruent and incongruent conditions at the same early time points. A concern with drawing inferences from a null result is that the failure to reject the null hypothesis in the neutral condition does not constitute evidence in favor of the null hypothesis. That is, perhaps the task-relevant information began to influence subjects' responses at the same points in time across all three conditions but that its influence was too weak in the neutral condition to detect with standard null-hypothesis tests. Weakly consistent with this possibility is the observation that the minimum stimulus-viewing duration was slightly less in the congruent condition, where both taskrelevant and irrelevant information can conspire together to yield an earlier response. Thus, to consider this possibility further, we calculated the Bayes Factor (Rouder, Speckman, Sun, Morey, \& Iverson, 2009) in the neutral condition at the same time points that the congruent and incongruent conditions first yielded reliable movements. The value of calculating the Bayes Factor is that it allows one to quantify the evidence in favor of the null hypothesis.

In Experiment 1, we used the trials from the seventh quantile to calculate the Bayes Factor in the neutral condition, as this was the quantile that yielded the first reliable result. This analysis revealed 4.4 to 1 odds in favor of the null hypothesis in the neutral condition. Looking at the eighth quantile (when the incongruent condition first yielded a reliable result), the Bayes Factor indicated 4.8 to 1 odds in favor of the null hypothesis. Thus, at these two early time points, the evidence is $\sim 4$ times greater for the null hypothesis in the neutral condition than it is for the alternative. In Experiment 2, we see largely the same pattern. Specifically, the Bayes Factor in the neutral condition at the seventh quantile, where the first reliable movements were observed, was 3.04. This indicates that the evidence was $\sim 3$ times greater for the null than the alternative at this time point. Looking at the eighth quantile, where the incongruent condition first yielded reliable movements, the Bayes Factor in the neutral condition indicated 1.85 to 1 odds in favor of the null hypothesis. Although this last result does not constitute strong evidence in favor of the null, the overall pattern of results is consistent across both experiments. Namely, at the same time points that the congruent and incongruent conditions first yielded reliable movements, the evidence indicates that the movements in the neutral condition were simply guesses as opposed to being controlled by the task-relevant information. This finding undermines the magnitude-difference explanation of the Simon effect, which holds that both the task-relevant and task-irrelevant information gain control of the response-formulation process at the same time (albeit to differing degrees).

\section{Earliest reliable movements reveal spatial capture}

According to the standard dual-route account of the Simon effect, the task-irrelevant attributes of the stimulus (i.e., its location) are encoded more rapidly than the task-relevant attributes (e.g., its color). In the standard version of the Simon task, where participants are asked to "respond as quickly and as accurately as possible," this assumption leads to the prediction that interference will be maximal across the earliest response latencies but then dissipate across the slower responses. But in a paradigm like ours, where subjects are required to initiate their responses in synchrony with an imperative go signal, this assumption leads to the prediction that there should be a stage of stimulus processing in which the stimulus-response transformation is controlled by the rapid encoding of task-irrelevant attributes followed by a transition to a subsequent stage in which the stimulus-response 
transformation is controlled by the slower encoding of task relevant attributes. The earlier findings by Buetti and Kerzel $(2008,2009)$ were largely consistent with this prediction insofar as the initial angle of their subjects' earliest responses was bimodal in the incongruent condition. That is, a small proportion of their subjects' earliest reaching responses were initially in the wrong direction. However, because the earliest responses in their studies already yielded a difference between congruent and incongruent trials, it was not clear from their findings when the task-relevant and task-irrelevant information first gained control of the response formulation process. Furthermore, because the largest proportion of the very earliest reaching responses in their studies were in the correct direction, it was not possible to distinguish between the time- and magnitude-difference accounts of the Simon effect.

The present study has addressed these shortcomings by coupling the reach-to-touch paradigm with the responsesignal procedure. To our knowledge, the present study is the first to demonstrate, with overt responses as the dependent measure, that the rapid encoding of task-irrelevant spatial attributes initially controls the stimulus-response transformation. Specifically, in both Experiment 1 and Experiment 2, the movements that were initiated within the first $\sim 100 \mathrm{~ms}$ of stimulus onset were not reliably correct or incorrect: they were guesses with a mean $\mathrm{x}$-velocity that was not different from zero. Soon afterwards the first reliable movements emerged, and these demonstrated a clear capture by the spatial position of the stimulus, with congruent trials eliciting correct movements and incongruent trials eliciting movements in the incorrect direction. Only later, after the first reliable movements emerged in the neutral condition, did the initial spatial capture give way to responses that were controlled by the taskrelevant attributes of the stimulus. This pattern of results follows directly from the assumptions of the dual-route models of the Simon effect but, again, to our knowledge, has not been reported previously with overt responses.

Although our results support the irrelevant information arriving before relevant information (i.e., the time-difference account) they do not rule out the possibility that there are also magnitude differences between relevant and irrelevant information. Indeed, our analysis of sequential effects suggests that they are best explained by a magnitude-difference rather than a time-difference account. We believe this intriguing finding deserves further research. One interpretation of it is that cognitive control triggered by the occurrence of conflict is mediated by magnitude rather than timing changes. That is control acts through a different mechanism than that which mediates the occurrence of interference in the first place.

Perhaps less obviously, we believe it is important to acknowledge that magnitude differences provide an alternative explanation to decay of irrelevant information for the eventual dominance of relevant information. This is illustrated in the right-most ("no decay") panel of Fig. 1, where the two types of information diverge at different times but both maintain their peak magnitude (i.e., the irrelevant information does not decay). However, because the peak is higher for the relevant information it eventually dominates the irrelevant information, and so the same biphasic shape occurs in the summed information as in the other accounts. We believe the latter observation should be acknowledged and examined more closely in future research; to the degree that a dependent measure reveals only the aggregated effects of relevant and irrelevant information, it cannot distinguish between decay and magnitude-difference accounts of the later time-course of the Simon effect, and indeed other interference effects.

\section{Conclusion}

The Simon effect is a unique phenomenon within cognitive psychology insofar as its magnitude is frequently observed to decrease with increasing response latencies. The opposite, where effect size increases with increasing response latencies, is the pattern that is normally observed in most other tasks. To explain this reverse pattern, contemporary accounts of the Simon effect appeal to dual-route architectures, which combine a fast, direct route that encodes the task-irrelevant spatial attributes with a slower, cognitive route that encodes the taskrelevant stimulus attributes. According to these models, the magnitude of the Simon-effect is thought to be a function of both the relative speed and strength of the two routes. The primary aim of the present study was to pit the relative speed aspect of this account against the relative strength aspect by determining whether activation from irrelevant information becomes available earlier than activation from relevant information, or whether they become available at the same time, but with the irrelevant information initially being stronger. Our results were most consistent with the relative speed (time-difference) account but inconsistent with the relative strength (magnitude-difference) account, except for a smaller magnitude-based modulation that occurred as a function of whether conflict was present on the last trial.

Acknowledgments This work was supported in part by a Future Fellowship to MF and a Professorial Fellowship to AH, both from the Australian Research Council. We would also like to acknowledge the contributions of Jason Friedman and Samantha Parker for their help in programming the experiments and in collecting the data, and helpful comments by Bernhard Hommel and an anonymous reviewer.

\section{References}

Atkeson, C. G., \& Hollerbach, J. M. (1985). Kinematic features of unrestrained vertical arm movements. Journal of Neuroscience, 5, 2318 2330

Bates, D. (2005). Fitting linear mixed models in R. R News, 5(1), 27-30. 
Botvinick, M. M., Braver, T. S., Barch, D. M., Carter, C. S., \& Cohen, J. D. (2001). Conflict monitoring and cognitive control. Psychological Review, 108(3), 624-652.

Buetti, S., \& Kerzel, D. (2008). Time course of the Simon effect in pointing movements for horizontal, vertical, and acoustic stimuli: Evidence for a common mechanism. Acta Psychologica, 129(3), 420-428.

Buetti, S., \& Kerzel, D. (2009). Conflicts during response selection affect response programming: Reactions toward the source of stimulation. Journal of Experimental Psychology: Human Perception and Performance, 35(3), 816.

Burle, B., van den Wildenberg, W., \& Ridderinkhof, K. R. (2005). Dynamics of facilitation and interference in cue-priming and Simon tasks. European Journal of Cognitive Psychology, 17(5), 619-641.

Cespón, J., Galdo-Álvarez, S., \& Díaz, F. (2012). The Simon effect modulates N2cc and LRP but not the N2pc component. International Journal of Psychophysiology, 84(2), 120-129.

Cohen, J. D., Dunbar, K., \& McClelland, J. L. (1990). On the control of automatic processes: A parallel distributed processing account of the Stroop effect. Psychological Review, 97(3), 332-361.

Craft, J. L., \& Simon, J. R. (1970). Processing symbolic information from a visual display: Interference from an irrelevant directional cue. Journal of Experimental Psychology, 83(3), 415-420.

De Jong, R., Liang, C.-C., \& Lauber, E. (1994). Conditional and unconditional automaticity: A dual-process model of effects of spatial stimulus-response correspondence. Journal of Experimental Psychology: Human Perception and Performance, 20(4), 731.

Finkbeiner, M., Coltheart, M., \& Coltheart, V. (2014). Pointing the way to new constraints on the dynamical claims of computational models. Journal of Experimental Psychology: Human Perception and Performance, 40(1), 172-185.

Forstmann, B. U., van den Wildenberg, W. P., \& Ridderinkhof, K. R. (2008). Neural mechanisms, temporal dynamics, and individual differences in interference control. Journal of Cognitive Neuroscience, 20(10), 1854-1865.

Freeman, J. B., Ambady, N., Rule, N. O., \& Johnson, K. L. (2008). Will a category cue attract you? Motor output reveals dynamic competition across person construal. Journal of Experimental Psychology: General, 137(4), 673.

Frith, C. D., \& Done, D. J. (1986). Routes to action in reaction time tasks. Psychological Research, 48, 169-177.

Ghez, C., Favilla, M., Ghilardi, M. F., Gordon, J., Bermejo, R., \& Pullman, S. (1997). Discrete and continuous planning of hand movements and isometric force trajectories. Experimental Brain Research, 115(2), 217-233.

Hasbroucq, T., \& Guiard, Y. (1991). Stimulus-response compatibility and the Simon effect: Toward a conceptual clarification. Journal of Experimental Psychology: Human Perception and Performance, 17(1), 246-266.

Hedge, A., \& Marsh, N. W. A. (1975). The effect of irrelevant spatial correspondence on two-choice response-time. Acta Psychologica, 39, 427-439.

Hilchey, M. D., Ivanoff, J., Taylor, T. L., \& Klein, R. M. (2011). Visualizing the temporal dynamics of spatial information processing responsible for the Simon effect and its amplification by inhibition of return. Acta Psychologica, 136(2), 235-244.

Hommel, B. (1993a). The relationship between stimulus processing and response selection in the Simon task: Evidence for a temporal overlap. Psychological Research, 55, 280-290.

Hommel, B. (1993b). Inverting the Simon effect by intention. Psychological Research, 55, 270-279.

Hommel, B. (1994a). Effects of irrelevant spatial S-R compatibility depend on stimulus complexity. Psychological Research, 56, 179184
Hommel, B. (1994b). Spontaneous decay of response-code activation. Psychological Research, 56(4), 261-268.

Hommel, B., Müsseler, J., Aschersleben, G., \& Prinz, W. (2001). The theory of event coding (TEC): A framework for perception and action planning. Behavioral and Brain Sciences, 24, 849-937.

Hommel, B., Proctor, R. W., \& Vu, K.-P. L. (2004). A feature-integration account of sequential effects in the Simon task. Psychological Research Psychologische Forschung, 68(1), 1-17.

Ivanoff, J. (2003). On spatial response code activation in a Simon task. Acta Psychologica, 112(2), 157-179.

Ivanoff, J., Blagdon, R., Feener, S., McNeil, M., \& Muir, P. H. (2014). On the temporal dynamics of spatial stimulus-response transfer between spatial incompatibility and Simon tasks. Frontiers in Neuroscience, 8, 1-17.

Ivanoff, J., Klein, R. M., \& Lupiáñez, J. (2002). Inhibition of return interacts with the Simon effect: An omnibus analysis and its implications. Perception \& Psychophysics, 64(2), 318-327.

Karayanidis, F., Provost, A., Brown, S. D., Paton, B., \& Heathcote, A. (2011). Switch-specific and general preparation map onto different ERP components in a task-switching paradigm. Psychophysiology, 48(4), 559-568.

Kello, C. T., \& Plaut, D. C. (2000). Strategic control in word reading: Evidence from speeded responding in the tempo-naming task. Journal of Experimental Psychology: Learning, Memory, and Cognition, 26, 719-750.

Kerzel, D., \& Buetti, S. (2012). Approach and avoidance movements are unaffected by cognitive conflict: A comparison of the Simon effect and stimulus-response compatibility. Psychonomic Bulletin \& Review, 19(3), 456-461.

Kliegl, R., Masson, M. E., \& Richter, E. M. (2010a). A linear mixed model analysis of masked repetition priming. Visual Cognition, 18(5), 655-681.

Kliegl, R., Wei, P., Dambacher, M., Yan, M., \& Zhou, X. (2010b). Experimental effects and individual differences in linear mixed models: Estimating the relationship between spatial, object, and attraction effects in visual attention. Frontiers in Psychology, 1, 238.

Koop, G. J., \& Johnson, J. G. (2011). Response dynamics: A new window on the decision process. Judgment and Decision Making, 6(8), 750-758.

Kornblum, S., Hasbroucq, T., \& Osman, A. (1990). Dimensional overlap: Cognitive basis for stimulus-response compatibility-A model and taxonomy. Psychological Review, 97, 253-270.

Kornblum, S. (1994). The way irrelevant dimensions are processed depends on what they overlap with: The case of Stroop and Simon-like stimuli. Psychological Research, 56, 130-135.

Kornblum, S., Stevens, G. T., Whipple, A., \& Requin, J. (1999). The effects of irrelevant stimuli: 1 . The time course of stimulus-stimulus and stimulus-response consistency effects with Stroop-like stimuli, Simon-like tasks, and their factorial combinations. Journal of Experimental Psychology: Human Perception and Performance, 25(3), 688-714.

Leuthold, H. (2011). The Simon effect in cognitive electrophysiology: A short review. Acta Psychologica, 136(2), 203-211.

Logan, G. D. (1980). Attention and automaticity in Stroop and priming tasks: Theory and data. Cognitive Psychology, 12(4), 523-553.

Lu, C. H., \& Proctor, R. W. (1994). Processing of an irrelevant location dimension as a function of the relevant stimulus dimension. Journal of Experimental Psychology: Human Perception and Performance, 20, 286-298.

Lu, C.-H., \& Proctor, R. W. (1995). The influence of irrelevant location information on performance: A review of the Simon and spatial Stroop effects. Psychonomic Bulletin \& Review, 2(2), 174-207.

MacLeod, C. M. (1991). Half a century of research on the Stroop effect: An integrative review. Psychological Bulletin, 109(2), 163.

Miller, E. K., \& Cohen, J. D. (2001). An integrative theory of prefrontal cortex function. Annual Review of Neuroscience, 24(1), 167-202. 
Mordkoff, T. (1998). The gating of irrelevant information in selectiveattention tasks [Abstract]. Abstracts of the Psychonomic Society, 3, 193

O'Leary, M. J., \& Barber, P. J. (1993). Interference effects in the Stroop and Simon paradigms. Journal of Experimental Psychology: Human Perception and Performance, 19(4), 830-844.

Praamstra, P. (2007). Do's and don'ts with lateralized event-related brain potentials. Journal of Experimental Psychology: Human Perception and Performance, 33(2), 497-502.

Pratte, M. S., Rouder, J. N., Morey, R. D., \& Feng, C. (2010). Exploring the differences in distributional properties between Stroop and Simon effects using delta plots. Attention, Perception, \& Psychophysics, 72(7), 2013-2025.

Proctor, R. W., Miles, J. D., \& Baroni, G. (2011). Reaction time distribution analysis of spatial correspondence effects. Psychonomic Bulletin \& Review, 18(2), 242-266.

Quek, G. L., \& Finkbeiner, M. (2013). Spatial and temporal attention modulate the early stages of face processing: Behavioural evidence from a reaching paradigm. PLOS ONE, 8(2), e57365.

Quek, G. L., \& Finkbeiner, M. (2014). Face-sex categorization is better above fixation than below: Evidence from the reach-to-touch paradigm. Cognitive, Affective, \& Behavioral Neuroscience, 1-13.

Reed, A. V. (1973). Speed-accuracy trade-off in recognition memory. Science, 181, 574-576.

Reed, A. V. (1976). List length and the time course of recognition in immediate memory. Memory \& Cognition, 4(1), 16-30.

Ridderinkhof, K. R. (2002a). Activation and suppression in conflict tasks: Empirical clarification through distributional analyses. In W. Prinz \& B. Hommel (Eds.), Common mechanisms in perception and action: Attention and performance XIX (pp. 494-519). New York: Oxford University Press.

Ridderinkhof, R. (2002b). Micro- and macro-adjustments of task set: activation and suppression in conflict tasks. Psychological Research, 66(4), 312-323.

Roswarski, T. W., \& Proctor, R. W. (1996). Multiple spatial codes and temporal overlap in choice-reaction tasks. Psychological Research, $59,196-211$.

Roswarski, T. E., \& Proctor, R. W. (2003). Intrahemispherical activation, visuomotor transmission, and the Simon effect: Comment on Wascher et al. (2001). Journal of Experimental Psychology: Human Perception and Performance, 29(1), 152-158.

Rouder, J. N., Speckman, P. L., Sun, D. C., Morey, R. D., \& Iverson, G. (2009). Bayesian $t$ tests for accepting and rejecting the null hypothesis. Psychonomic Bulletin \& Review, 16(2), 225-237.

Sanders, A. F. (1967). Some aspects of reaction processes. Acta Psychologica, 27, 115-130.

Simon, J. R., \& Acosta, E. (1982). Effects of irrelevant information on the processing of relevant information: Facilitation and/or interference? The influence of experimental design. Perception \& Psychophysics, 31, 383-388.

Simon, J. R., Acosta, E., Mewaldt, S. P., \& Speidel, C. R. (1976). The effect of an irrelevant directional cue on choice reaction time: Duration of the phenomenon and its relation to stages of processing. Perception \& Psychophysics, 19(1), 16-22.

Simon, J. R., \& Craft, J. L. (1970). Effects of an irrelevant auditory stimulus on visual choice reaction time. Journal of Experimental Psychology, 86, 272-274.
Simon, J. R., \& Rudell, A. P. (1967). Auditory S-R compatibility: The effect of an irrelevant cue on information processing. Journal of Applied Psychology, 51, 300-304.

Simon, J. R., \& Small, A. M., Jr. (1969). Processing auditory information: Interference from an irrelevant cue. Journal of Applied Psychology, $53,433-435$

Smit, A. C., \& van Gisbergen, J. A. (1990). An analysis of curvature in fast and slow human saccades. Experimental Brain Research, 81(2), 335-345.

Speckman, P. L., Rouder, J. N., Morey, R. D., \& Pratte, M. S. (2008). Delta plots and coherent distribution ordering. The American Statistician, 62(3), 262-266.

Stroop, J. R. (1935). Studies of interference in serial verbal reactions. Journal of Experimental Psychology, 18, 643-661.

Stürmer, B., Leuthold, H., Soetens, E., Schröter, H., \& Sommer, W. (2002). Control over location-based response activation in the Simon task: Behavioral and electrophysiological evidence. Journal of Experimental Psychology: Human Perception and Performance, 28(6), 1345-1363.

Stürmer, B., Leuthold, H., \& Sommer, W. (1998). Strategische Effekte und kurzfristige Modulationen im Simon-Paradigma [Strategic effects and short-term modulations in the Simon paradigm] [Abstract]. In H. Lachnit, A. Jacobs, \& F. Rosler (Eds.), Experimentelle Psychologie: Abstracts der 40. Tagung experimentell arbeitender Psychologen (pp. 365-366). Lengerich: Pabst Science Publishers.

Tagliabue, M., Zorzi, M., Umilta, C., \& Bassignani, F. (2000). The role of long-term-memory and short-term-memory links in the Simon effect. Journal of Experimental Psychology: Human Perception and Performance, 26(2), 648-670.

Taylor, T. L., \& Klein, R. M. (1998). On the causes and effects of inhibition of return. Psychonomic Bulletin \& Review, 5, 625-643.

Umilta, C., \& Zorzi, M. (1997). Commentary on Barber and O'Leary: Learning and attention in S-R compatibility. In B. Hommel \& W. Prinz (Eds.), Theoretical issues in stimulus-response compatibility (pp. 173-178). Amsterdam: North-Holland.

Valle-Inclán, F. (1996). The locus of interference in the Simon effect: An ERP study. Biological Psychology, 43(2), 147-162.

Vallesi, A., \& Umiltà, C. A. (2009). Decay of stimulus spatial code in horizontal and vertical Simon tasks. The Journal of General Psychology, 136(4), 350-373.

Wascher, E., Schatz, U., Kuder, T., \& Verleger, R. (2001). Validity and boundary conditions of automatic response activation in the Simon task. Journal of Experimental Psychology: Human Perception and Performance, 27, 731-751.

Wiegand, K., \& Wascher, E. (2005). Dynamic aspects of stimulus-response correspondence: Evidence for two mechanisms involved in the Simon effect. Journal of Experimental Psychology: Human Perception and Performance, 31, 453-464.

Wiegand, K., \& Wascher, E. (2007). Response coding in the Simon task. Psychological Research, 71(4), 401-410.

Woestenburg, J. C., Verbaten, M. N., Van Hees, H. H., \& Slangen, J. L. (1983). Single trial ERP estimation in the frequency domain using orthogonal polynomial trend analysis (OPTA): Estimation of individual habituation. Biological Psychology, 17, 173-191.

Zhang, J., \& Kornblum, S. (1997). Distributional analysis and De Jong, Liang, and Lauber's (1994) dual-process model of the Simon effect. Journal of Experimental Psychology: Human Perception and Performance, 23(5), 1543-1551. 